\title{
A Two-photon Fluorescence Autocorrelator for a Nd:YLF Modelocked Laser
}

by

Peter Alexander Kner
DOE/ER/ $14012--T 3$

DES2 040666

Sulumitted to the Department of Electrical Engineering and Computer Science in partial fulfillment of the requirements for the degrees of Bachelor of Science in Electrical Science and Engineering

and

Bachelor of Science in Physics

at the

MASSACHUSETTS INSTITUTE OF TECHNOLOGY

May 1991

Copyright Peter Alexander Kner, 1991

The author hereby grants to MIT permission to reproduce and to distribute copies of this thesis document in whole or in part.

$$
\text { Fo } 02-89 E R / 40 / 2
$$

Author.

Department of Electrical Engineering and Computer Science May 17, 1991

Certified by

Peter L. Hagelstein
Professor

Thesis Supervisor

Accepted by

Leonard A. Gould

Chairman. Department Committee on Undergraduate Theses 


\title{
A Two-photan Fluorescence Autocorrelator for a Nd:YLF Modelocked Laser \\ by
}

Peter Alexander Kner

\author{
Submitted to the Department of Electrical Engineering and Computer Science \\ on May 17, 1991, in partial fulfillment of the \\ requirements for the degrees of \\ Bachelor of Science in Electrical Science and Engineering \\ and \\ Bachelor of Science in Physics
}

\begin{abstract}
In this thesis, I discuss the deisign and implementation of an autocorrelator for an astively modelocked Nd:YLF laser at wavelength $1.054 \mu \mathrm{m}$. A dye is used to generate a broadband two-photon fluorescence (TPF) signal at $570 \mathrm{~nm}$ which is the autocorrelation of the laser pulses. Two different technigues are discussed. A colliding pulse scheme can be used to generate a TPF autocorrelaition signal as a function of distance, or an interferometer technique can be used to generaie an autocorrelation signal as a function of the delay in an interferometer arm. Experimental results are discussed, but they are inconclusive because of difficulties in interpreting the signal.
\end{abstract}

Thesis Supervisor: Peter L. Hagelstein

Title: Professor

\section{DISCLAIMER}

\begin{abstract}
This report was prepared as an account of work sponsored by an agency of the United States Government. Neither the United States Fovernment nor any agency thereof, nor any of their employees, makes any warranty, express or implied, or assumes any legal liability or responsi. bility for the accuracy, completeness, or usefulness of any information, apparatus, product, or process disclosed, or represents that its use would not infringe privately owned rights. Reference herein to any specific commercial product, process, or service by trade name, tradernark, manufacturer, or otherwise does not necessarily constitute or imply its endorsement, recommendation, or favoring by the United States Government or any agency thereof. The views and opinions of authors expressed herein do not necessarily state or reflect those of the Unitad States Government or any agency theroof.
\end{abstract}

The Goverment reserves for itself and others acting on its behalf a royalty free, nonexclusive, irrevocable, world-wide license for govermental purposes to publish, distribute, translate, duplicate, exhibit, and perform any such data copyrighted by the contractor. 


\section{Contents}

1 Introduction 8

2 Autocorrelator Theory 11

2.1 Quantum Theory of Two-Photon Absorption . . . . . . . . . . . 11

2.2 Plane Wave Model . . . . . . . . . . . . . . . . . . 16

2.2 .1 Basic Anaiysis . . . . . . . . . . . . . . 16

2.2 .2 Considering Bearn Attenuation ............. 18

2.3 Gaussian Beam Model . . . . . . . . . . . . . . . . . 21

2.4 Copropagating Pulses . . . . . . . . . . . . . . . . 22

2.5 The Autocorrelation Sigral . . . . . . . . . . . . . . . 24

2.6 Imperfect Beam Overlap $\ldots \ldots \ldots \ldots 24$

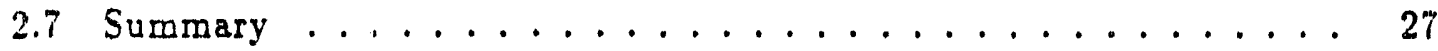

3 Design 28

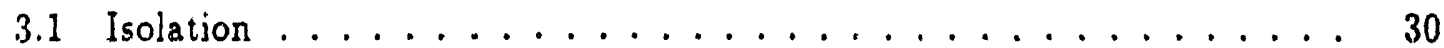

3.2 Copropagating Puls= Design . . . . . . . . . . . . . . 34

4 Imagiug and Detection 36

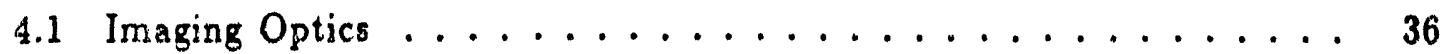

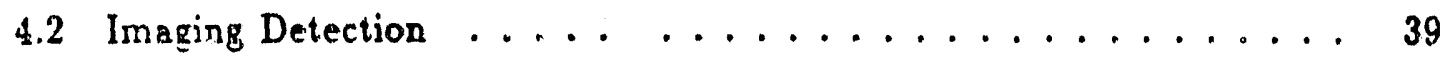

4.2 .1 Reticon Triggering .................. 41

4.3 Total Fluorescerce Detection . . . . . . . . . . . . . . . . 42

4.3 .1 Normalization .................... 44 
5 Experimental Results

6 Summary and Conelusions

A Optical design and circuit diagram for section 4.3.1 


\section{List of Figures}

2.1 Two-photon Fluorescence $\ldots \ldots \ldots \ldots \ldots \ldots \ldots$

2.2 Absorption (solid line) and fluorescence (dashed line) spectra for Rho. damine $6 \mathrm{G}$ in an ethanol solution $\ldots \ldots \ldots \ldots \ldots 16$

2.3 The basic setup for measuring the autocorrelation of a pulse using two-photon fluorescence $\ldots \ldots \ldots \ldots \ldots \ldots$

2.4 The fluorescence signal, $S(r)$, assuming a Gaussian pulse shape. The $y$-axis is normalized to $I_{1}$. The $x$-axis is in centimeters from the center of the cell. For the largest curve, $I_{2}=I_{1}$ and there is not attenuation through the dye. For the second curve $I_{2}$ is $80 \%$ of $I_{1}$ and the total attenuation through the cell is $5 \%$. For the third curve $I_{2}$ is half of $I_{1}$,

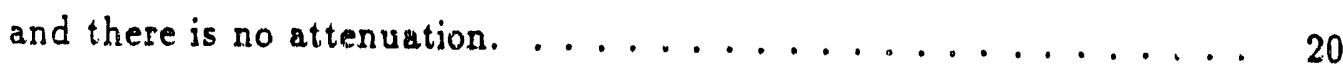

2.5 Co-propagating pulse autocorrelation $\ldots \ldots \ldots \ldots \ldots$

2.6 The autocorrelation signal of a non-modelocked laser . . . . . 25

2.7 The autocorrelation signal of a burst of noise noise . . . . . . 25

3.150 percent bearn splitter design $\ldots \ldots \ldots \ldots \ldots \ldots$

3.2 The pulse is reflected back on itself in the dye so that the beginning of the pulse is autocorrelated with the end . . . . . . . . . . 29

3.3 Each pulse is autocorrelated with the next pulse in the train. . . . 30

$3-4$ Optical Isolator . . . . . . . . . . . . . 32

$3-5$ Isolation by beam misalignment $\ldots \ldots \ldots \ldots \ldots \ldots \ldots$

3.6 Autocorrelator using TPF and a Michelson Interferometer . . . . 35

4-1 Calculating the collection efficiency of imaging optics . . . . . 37 
4.2 Imaging Optics (not to scale) ................ 38

4-3 A circuit to create a trigger signal for the oscilloscope . . . . . . . . 42

4.4 Photodiode Circuit .................... 43

4-5 Focusing the Fluorescence onto a Single Photodiode . . . . . . . . . 44

4-6 A design for electronically normalizing the measured Aluorescence signal 46

5.1 Autocorrelator Design used . . . . . . . . . . . . . . 48

5.2 oscilloscope trace of array signal with laser Q-switched and modelocked (vertical $10 \mathrm{mV} / \mathrm{div}$, horizontal $1 \mathrm{~ms} / \mathrm{div}$ ) . . . . . . . . . 49

5.3 oscilloscope trace of array signal with laser neither Q-switched nor modelocked (vertical $10 \mathrm{mV} /$ div, horizontal $1 \mathrm{~ms} / \mathrm{div}$ ) ....... 50

5.4 fiuorescence trace with the beam blocked (vertical $20 \mathrm{mV} /$ div, horizon.

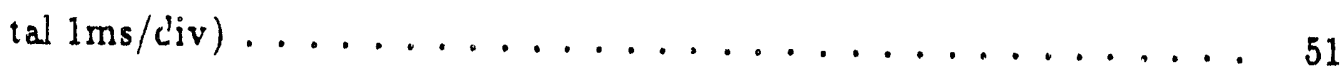

5.5 fluorescence trace with the beam unblocked (vertical $20 \mathrm{mV} / \mathrm{div}$, hori-

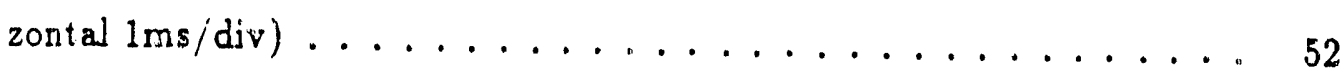

5.6 fiuorescence trace with the beam blocked (vertical $20 \mathrm{mV} /$ div, horizontal $1 \mathrm{~ms} / \mathrm{div}) \ldots \ldots \ldots \ldots \ldots \ldots \ldots \ldots \ldots \ldots$

5-i fluorescence trace with the beam unblocked (vertical $20 \mathrm{mV} / \mathrm{div}$, horizontal lins/div $\ldots \ldots \ldots \ldots \ldots \ldots$

5.8 Comparison of the predicted contrast ratio to the measured contrast ratio as a function of the distance from the end mirror to the fluorescence: curve 1 is the case with equal beam intensities and no attenuation, curve 2 assumes that the intensity of the returning pulse is $80 \%$ that of the incoming pulse, $I_{1}$, and curve 3 accounts for beam divergence as well. 


\section{List of Tables}

2.1 two photon fuorescence cross-sections . . . . . . . . . 15

4.1 K series EG\&G Reticon diode array (data from the 1989 Image Sensing Products Databook) ..................... 39

4.2 Logic Table for a J-K flip-flop . . . . . . . . . . . . . . 41

4.3 United Technologies PIN-10D diode characteristics . . . . . . 42 


\section{Chapter 1}

\section{Introduction}

The XRL group at MIT will use a Q-switched modelocked ND:YLF laser in their $X$-ray laser experiments. It is important to know the temporal width of the pulses in order to evaluate the experimental results.

The nominal pulse width of the ND:YLF laser is $100 \mathrm{ps}$, which cannot be measured by conventional electronics because electronic time constants are too large. Short optical pulses are traditionally measured by autocorrelation techniques. Provided that the general shape of the pulse is known, the autocorrelation of the pulse intensity, which is the convolution of the pulse with itself, provides a measurement of the pulse width. Second-harmonic generation (SHG) and two-photon fluorescence (TPF) are the frocesses commonly used to generate the autocorrelation signal.

SHG autocorrelation was first proposed in 1966 [3]. The output of a Michelson interferometer is focused into a nonlinear crystal which generates the second-harmonic. As the Michelson interferometer is scanned, the autocorrelation of the pulse intensity is mapped by the second-harmonic intensity [3] [5] [6].

TPF autocorrelation techniques were also developed in the late 1960s and have been used extensively since then [3] [4]. A dye dissolved in an alcoholic solution is the fluorescent material, and the autocorrelation signal is obtained by splitting a pulse in half and passing the two halves through each other in a glass cell containing the dye solution. The autocorrelation is then given by the fluorescence intensity as a function of position along the cell. 
The design and implementation of a TPF autocorrelator for the ND:YLF laser was begun in December 1989 by Rolf Wyss [10]. The project was not completed because of problems with the choice of beam intensity and dye concentration. In this paper, I review the theory of TPF autocorrelators and explore different designs in more depth. Then I discuss the results that I obtained which, also, were not conclusive.

TPF autocorrelators consist of two distinct sections. One section consists of the dye cell and the optics for passing the pulses through the cell. An important con. sideration in the design of this section is feedback into the laser. When the laser is amplified by an amplifier external to the laser cavity, feedback can damage the laser rod. Alignment is another important consideration. The magnitude of the autocorrelation signal depends on the amount of overlap between the two pulses in the dye cell. Because the pulses are focused to small diameter in the dye cell, the alignment of the pulses is critical. An effective technique for aligning the optics is needed.

The other section of the autocorrelator is the system for imaging and recording the fluorescence, which is the autocorrelation signal. The imaging optics must collect as much of the fluorescence as possible so that the signal can be recorded. In most designs, the signal is recorded by film [3] [4] or video [9]. Film is a very sensitive and the signal can be accumulated over as many pulses as necessary. Unfortunately film requires time and equipment to develop. It would be nice have the results immediately. In this paper a design to image the signal onto a diode array is evaluated [10]. Again alignment is critical because the fluorescence streak and the diode array are both very narrow.

The low repetition rate of the ND:YLF laser makes alignment more difficult. (The laser flash lamp is not run continuously but is pulsed.) The first section mentioned above can be aligned effectively with a Helium-Neon laser, but the second section can only be aligned with the ND:YLF because the imaging optics must be aligned to the fluorescence.

In practice, aligning the imaging optics was difficult because of the low repetition rate and the small amount of fluorescence. I performed experiments with a $0.01 \mathrm{M}$ solution of Rhodamine $6 \mathrm{G}$ in ethanol and got experimental results using the diode 
array without any optics. These results are inconclusive because they did not exhibit a large enough contrast ratio between the fluorescence of maximum pulse overlap at the center of the cell and the fluorescence of no pulse overlap. Many authors have not found the 3 to 1 contrast ratio of an ideal TPF experiment [3] [9] [1], but some of the contrast ratios that I measured were low enough to be due to noise. It is difficult to determine the reason for the low contrast ratio because the alignment of the different sections is important and the low contrast might be due to the laser itself. Although film is difficult to use, it would be helpful in setting up and diagnosing the problems of a TPF autocorrelator.

At the present time, the laser is out of commission because of damage to the laser rod. The next experiments will use a Michelson interferometer design similar to the SHG autocorrelator configurations, because this design should be more sensitive and easier to align. 


\section{Autocorrelator Theory}

Optical autocorrelators work on the principle of mapping the temporal characteristics of the light pulses onto the spatial domain. This is convenient and accurate because even very short pulses which cannot be resolved by electronics map onto easily measurable distances; light travels $0.3 \mathrm{~mm}$ in 1 ps.

The use of two-photon fluorescence (TPF) to measure the temporal width of pulses has been well explored in the literature [4] [9]. This design is based on initial work done by Rolf Wyss in collaboration with the MIT XRL group in January 1990. In this chapter I will describe the theory behind measuring autocorrelation with TPF.

\subsection{Quantum Theory of Two-Photon Absorption}

The physical process of two-photon fluorescence involves the excitation of a molecule to a higher state by the absorption of two photons at frequency $\omega$. A photon is then spontaneously emitted at a frequency slightly less than $2 \omega$ when the molecule radiatively decays. (Some energy is lost by processes other than photon emission.) Figure 2-1 illustrates this process.

1 will outline the theory of two-photon absorption and relaxation for a simple 3 level system, to relate the macroscopic description of TPF, to the basic physics. This derivation follows that of Pantell, [7].

The power absorbed from the field is 


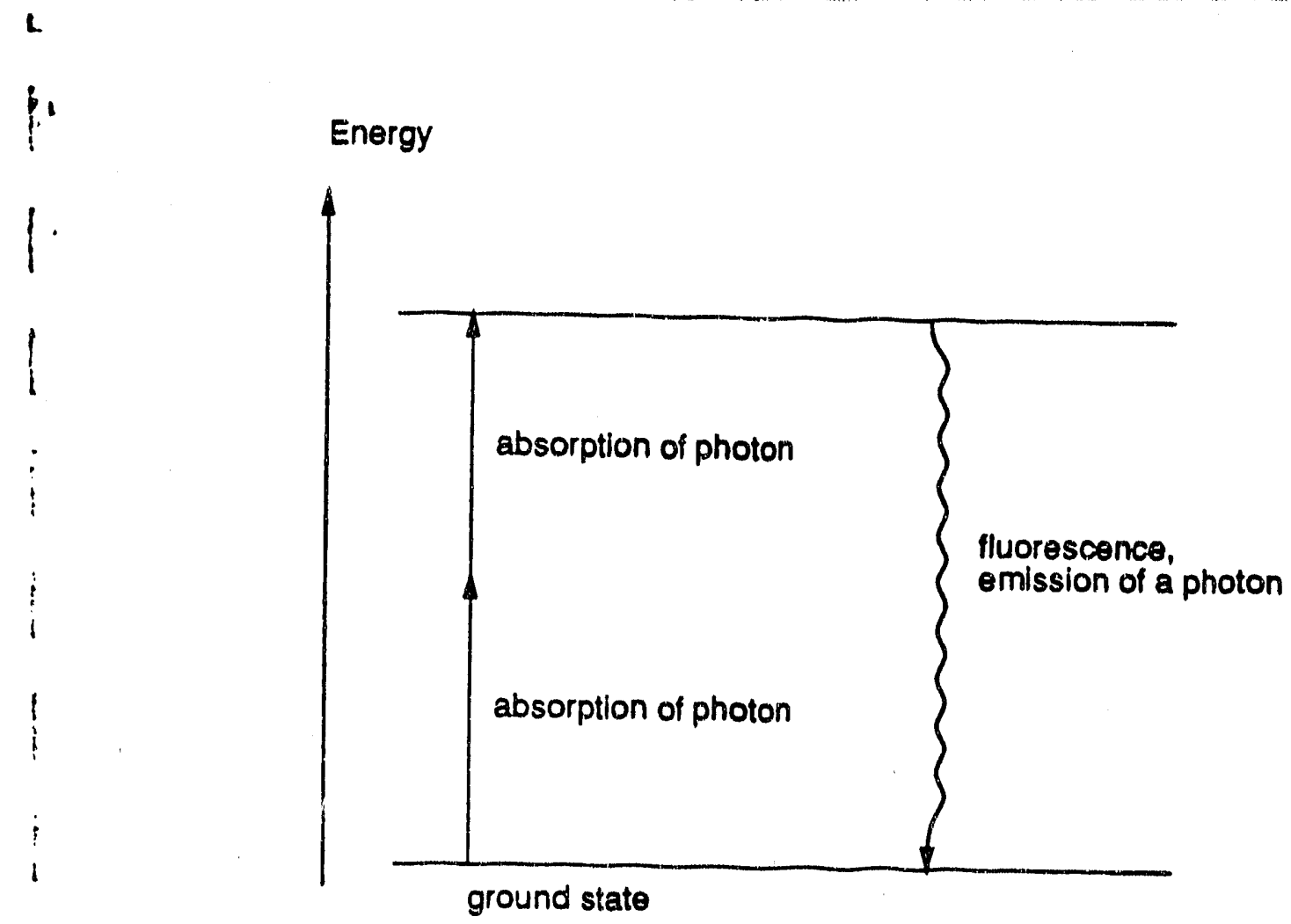

Figure 2-1: Two-photon Fluorescence

$$
P=\hbar \omega_{21} N \Gamma
$$

where $\omega_{21}$ is the frequency of the transition made by the molecule, $N$ is the number of molecules per unit volume, and $\Gamma$ is the transition probability for two-photon absorption. $I$ is given by the equation,

$$
\Gamma=\left\langle\frac{\partial \rho_{22}}{\partial t}\right\rangle
$$

where $\rho$ is the density matrix and the bar denotes time averaging over the period of electromagnetic wave.

The equation of motion for the density matrix is

$$
i \hbar \frac{\partial \rho}{\partial t}=[H, \rho]
$$

The Hamiltonian is the Hamiltonian of the molecule plus a perturbation due to the 
dipole interaction of the molecule with the electric field.

$$
H=H_{0}+H^{\prime}
$$

The matrix elements of $H_{0}$ are defined as

$$
\left[H_{0}\right]_{i j}=E_{i} \delta_{i j}
$$

The matrix elements of $H^{\prime}$ are defised as

$$
H_{i j}^{\prime}=\vec{\mu}_{i j} \cdot \vec{E}(t)
$$

For this jerivation, it is assumed that the siates have definite parity, so that $H_{i i}^{\prime}=0$. In this formula the electric field is taken to be

$$
\vec{E}(t)=\vec{E} e^{-i \omega t}+\vec{E}^{*} e^{i \omega t}
$$

Because we are interested in two-photon absorption, it is assumed that the frequency of this electric field is $\omega \approx \frac{1}{3} \omega_{31}$.

Equation 2.3 is solved using perturbation theory. It is assumed that the system starts completely in the ground state, so that the zeroth order solution, $\rho^{(0)}$, is $\rho_{11}^{(0)}=1$ and all the other zeroth order matrix elements are zero. The perturbation equations are

$$
i \hbar \cdot \frac{\partial \rho_{i i}^{(n)}}{\partial t}=\left[H^{\prime}, \rho^{(n-1)}\right]_{i i}
$$

and, for $i=j$,

$$
i \hbar\left(\frac{\partial}{\partial t}+i \omega_{i j}+\frac{1}{T_{i j}}\right) p_{i j}^{(n)}=\left[H^{\prime}, \rho^{(n-1)}\right)_{i j} .
$$

$r_{1 j}$ is the reiaxation time between states $i$ and $j$, and $w_{i j}$ is the frequency of their energy difference.

I will not solve the equations, but will only quote the results [7]. The transition 
rate for single photon absorption is found to be the time derivative of the second order term, $\left(\frac{\partial g_{27}^{(2)}}{\partial t}\right)$, which bas the resonance $\left(i\left(j-\omega_{12}\right)+1 / r_{32}\right)^{-1}$, indicating single-photon absorption. The single-photon absorption rate is

$$
\Gamma_{(1)}=\frac{1 / \tau_{12}}{\left(\omega-\omega_{12}\right)^{2}+1 / \tau_{12}^{2}} \frac{\left|\vec{\mu}_{12} \cdot \vec{E}\right|^{2}}{2 \hbar^{2}}
$$

The two-photon absorption transition rate is

$$
\begin{gathered}
\Gamma_{(2)}=\left\langle\frac{\partial \rho_{22}^{(4)}}{\partial t}\right\rangle \\
\Gamma_{(2)}=\frac{1 / \tau_{12}}{8 \hbar^{4}\left(\left(2 \omega-\omega_{12}\right)^{2}+1 / \tau_{12}^{2}\right)} \frac{\left|\vec{\mu}_{13} \cdot \vec{E}\right|^{2}\left|\vec{\mu}_{23} \cdot \vec{E}\right|^{2}}{\left(\omega-\omega_{31}\right)^{2}}
\end{gathered}
$$

In an isotropic medium, such as a dye solution, the dipole moments will be in all different direcions, and the average dipole moment can be used. Because all dipole moment dicections will be equally likely, the square of the dipols moment, $\left|\vec{\mu}_{12} \cdot \vec{E}\right|^{2}$, can te replaced with its average, $\frac{1}{3}\left|\mu_{12}\right|^{2}|E|^{2}[7]$. A term, $L^{2}$, is also added to correct for the difference berween the local field seen by a molecule and the nacroscopic field. This term is called the Lorentz correction factor [7] and is due to polarization of the field by surrounding molecules. $L=\left[\left(n^{2}+2\right) / 3\right]^{2}$. Thus, equation 2.12 can be writ.en

$$
\Gamma_{(2)}=\frac{1 / \tau_{12}}{8 \hbar^{4}\left(\left(2 \omega-\omega_{12}\right)^{2}+1 / \tau_{13}^{2}\right)} \frac{L^{2}\left|\mu_{33}\right|^{2}\left|\mu_{23}\right|^{2}|\vec{E}|^{4}}{9\left(\omega-\omega_{31}\right)^{2}}
$$

The transition rate, and hence the power absorpition densiy, is proportional to the square of the intensity, $|E|^{4}$. This leads to the impu: tant macroscopic description of TPF: the absorbed radiation will be reradiated as fluorescence at frequency $2 \omega$, so an equation for $P_{f}$, the power density of emitted fluorescence radiation can be written:

$$
P_{f}=q_{f}\left(\frac{\omega_{f}}{2 \omega}\right) \hbar \omega, N \Gamma_{(2)}=\alpha I_{\omega}^{2} .
$$

where $I_{\omega}$ is the intensity at the pulse wavelength (1054nm for the ND:YLF) and $q_{f}\left(\frac{w_{f}}{2 \omega}\right)$ is the fuorescence efficiency. The constant $\alpha$ is also written as 


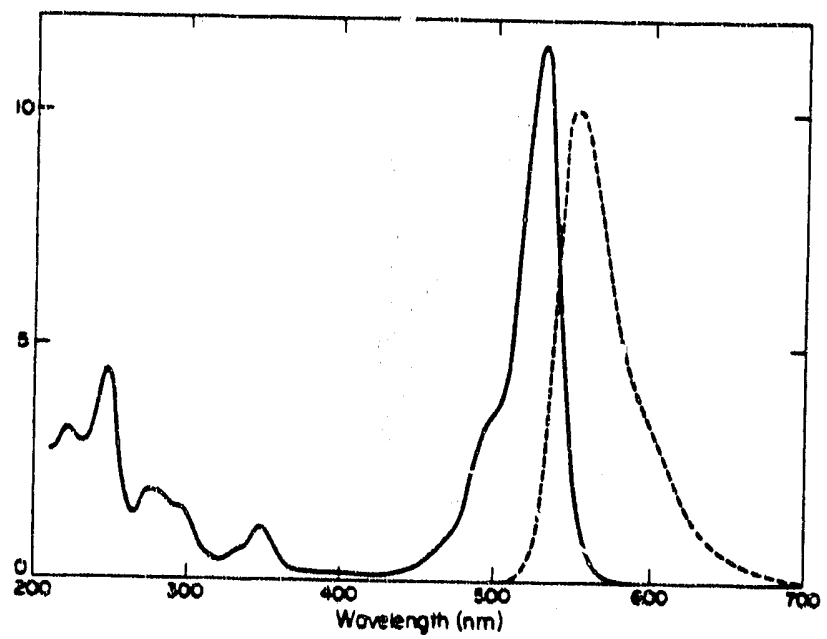

Figure 2-2: Absorption (solid line) and flusrescence (dashed line) spectra for Rhodamine $6 \mathrm{G}$ in an ethanol solution

with itself in the dye. The intensity of the fluorescence signal is the autocorrelation of the pulse is a function of space. Figure 2-3 is a diagram of a general autocorrelator setup. In the next sections I will describe the plane war model for the pulse electromagnetic field, then I will discuss approximatiag the Gussian beam model.

\subsection{Plane Wave Model}

\subsubsection{Basic Analysis}

If we treat the pulses as plane waves, then the electric field in the dye is

$$
\vec{E}=E(t) \hat{x}
$$

The choice electric field polarization in the $x$ direction is arbitrary. $E(t)$ is the sum of two pulses at frequency $\omega$ traveling in opposite directions.

$$
E(t)=E_{1} A\left(t-\frac{n\left(z+z_{0}\right)}{c}\right) e^{i(k z-\omega t)}+E_{2} A\left(t+\frac{n\left(z-z_{0}\right)}{c}\right) e^{i(k z+\omega t)}+c . c .
$$

where $A(t)$ is the normalized pulse shape, $\int_{-\infty}^{+\infty} A^{2} d t=1 ; E_{1}$ is the electris field of the 
pulse entering from the left and $E_{2}$ is the electric field of the pulse entering from the right. For the setup of figure $2-3, E_{1}$ should equal $E_{2}$. The time average intensity at frequency $\omega$ is

$$
I_{\omega}=\epsilon_{\circ} n c\left\langle|E|^{2}\right\rangle
$$

Substituting equation 2.17 into equation 2.18

$I_{\omega}=\epsilon_{0} n c E_{1}^{2} A^{2}\left(t-\frac{n z}{c}\right)+\epsilon_{\circ} n c E_{2}^{2} A^{2}\left(t-\frac{n z}{c}\right)+2 \epsilon_{0} n c E_{1} E_{2} A\left(t-\frac{n z}{c}\right) A\left(t+\frac{n z}{2}\right) \cos (2 k z)$

A simpler way of writing equation 2.19 is

$$
I_{\omega}=I_{1}+I_{2}+2 \sqrt{I_{1} I_{2}} \cos 2 k z
$$

where $I_{1}$ and $I_{2}$ are defined by the equality of equations 2.19 and 2.20 . We use equation 2.14 to find the fluorescence power density

$$
P_{f}=\alpha\left(I_{1}^{2}+I_{2}^{2}+2 I_{1} I_{2}\left(1+2 \cos ^{2} 2 k z\right)+2\left(I_{1}+I_{2}\right) \sqrt{I_{1} I_{2}} \cos 2 k z\right) .
$$

$P_{f}$ has a rapid spatial variation which will not be resolved by film or diode arrays so we average over spatial variations on the order of $\lambda$.

$$
P_{f}=\alpha\left(I_{1}^{2}+I_{2}^{2}+4 I_{1} I_{2}\right)
$$

The signal recorded by a detector will be the time integral of the fluorescence signal because of persistence in the dye and because of the limited detector bandwidth. We will also integrate over the cross section of the dye because we are not interested in variation in those dimensions and the detector will effectively integrate over them. Thus the recorded signal will be, $S\left(\frac{2 n z}{c}\right)=\iiint_{-\infty}^{+\infty} P_{f} d t d x d y$. 


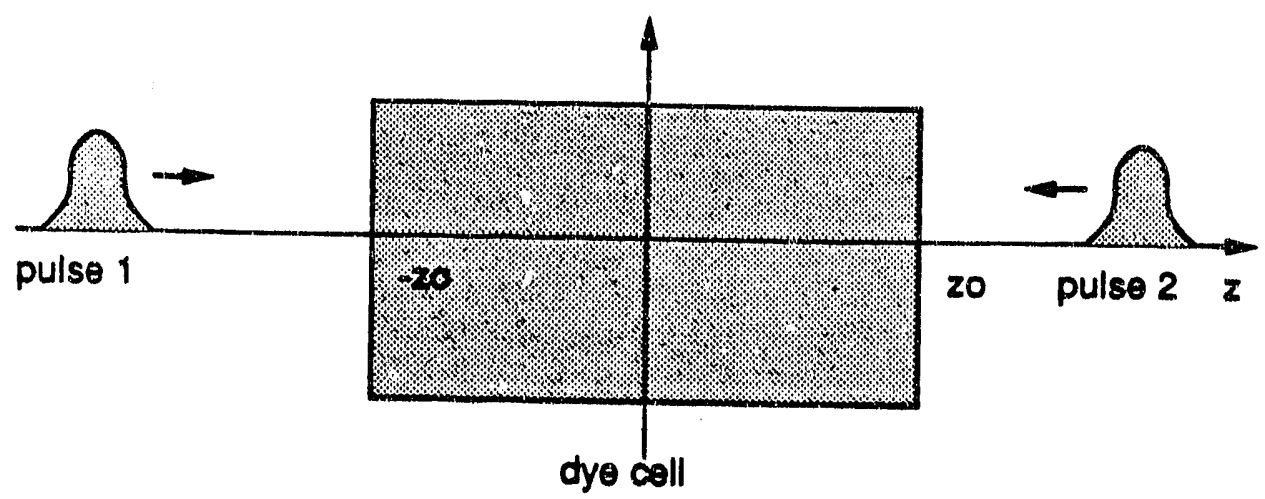

Figure 2-3: The basic setup for measuring the autocorrelation of a pulse using two. photon fluorescence

$$
\begin{gathered}
S(\tau)=\alpha \iint d x d y \int_{-\infty}^{+\infty} d t\left(I_{1}^{2}+I_{2}^{2}+4 I_{1} I_{2}\right) \\
S(\tau)=\alpha \iint d x i y \int_{-\infty}^{+\infty} d t\left(I_{1}^{2}+I_{2}^{2}\right)[1+4 G(\tau)],
\end{gathered}
$$

where $\tau=\frac{2 \pi z}{c}$ and $G(\tau)$ is the normalized autocorrelation function,

$$
G(\tau)=\frac{\int_{-\infty}^{+\infty} I_{1}(t) I_{2}(t-\tau) d t}{\int_{-\infty}^{+\infty}\left(I_{1}^{2}+I_{2}^{2}\right) d t}
$$

Thus, the fluorescence signal at $\omega_{f}$ yields the autocorrelation of the pulse at $1054 \mathrm{~nm}$ for the configuration of figure 2-3. Figure 2-4 is a graph of $S(\tau)$ for a Gaussian pulse. (The two small curves on figure $2-4$ will be discussed in the next section.)

\subsubsection{Considering Beam Attenuatiun}

The preceding analysis assumes that as the beam goes through the dye cell it is not appreciably attenuated by two-photon absorption or any other process. But of course the beam is going to be attenuated by two-photon absorption and we should consider the case when attenuation is not negligible. Figure 2-2 indicates that single-photon absorption will be low at the ND:YLF wavelength, $1054 \mathrm{~nm}$, so I will ignore the effects 
of single-photon absorption. I will only consider the case of weak attenuation because if the attenuation is strong, the fluorescence signal will not give any information about the autocorrelation function of the pulse.

The beam attenuaticn is given by the equation 2.14 because the energy emitted as fluorescence is energy absorbed from the beam:

$$
\begin{gathered}
\frac{\partial I}{\partial z}=-\gamma I^{2}, \\
I=\frac{I_{0}}{1+I_{0} \gamma z} \approx I_{0}\left(1-I_{0} \gamma z\right),
\end{gathered}
$$

where $\gamma=\frac{N o}{n_{\omega}}$ and $I_{0}$ is the pulse intensity upon entering the dye cell. The constant $\alpha$ in equation 2.14 is not the same as $\gamma$ in equation 2.26 because some of the energy absorbed is not emitted as fluorescence. $q_{f}\left(\frac{\omega_{f}}{2 \omega}\right)$, the quantum efficiency, in equation 2.15 accounts for this. We can substitute for $I_{1}$ and $I_{2}$ using equation 2.27. Thus,

$$
I_{1}=I_{01}\left(\mathbf{i}-I_{01} \gamma z\right)
$$

where $I_{01}=\frac{1}{2} \epsilon_{o} n c E_{1}^{2} A^{2}\left(t-\frac{n z}{c}\right)$ is the intensity incident on the dye cell in pulse 1 , and $I_{2}$ is similarly defined. We can now rewrite equation 2.22 accounting for beam attenuation. To first order,

$$
\begin{gathered}
P_{f}=\alpha\left(I_{01}^{2}+I_{02}^{2}+4 I_{01} I_{02}\right)- \\
-\alpha\left(2 I_{01}^{2}\left(I_{01} \gamma\right)\left(z+z_{0}\right)+2 I_{02}^{2}\left(I_{02} \gamma\right)\left(z_{0}-z\right)+4 I_{01} I_{02}\left(\left(I_{01}+I_{02}\right) \gamma z_{0}-\left(I_{01}-I_{02}\right) \gamma z\right)\right.
\end{gathered}
$$

Thus, if the pulses are the same intensity, the signal will be the same shape, only smaller. If the pulses have unequal intensities, the signal will be skewed. Figure 2-4 is a graph of $S(\tau)$, equation 2.24, assuming a Gaussian pulse shape and different attenuations and relative intensities. Notice also that if the puises have unequal intensities, the contrast ratio will be reduced. 


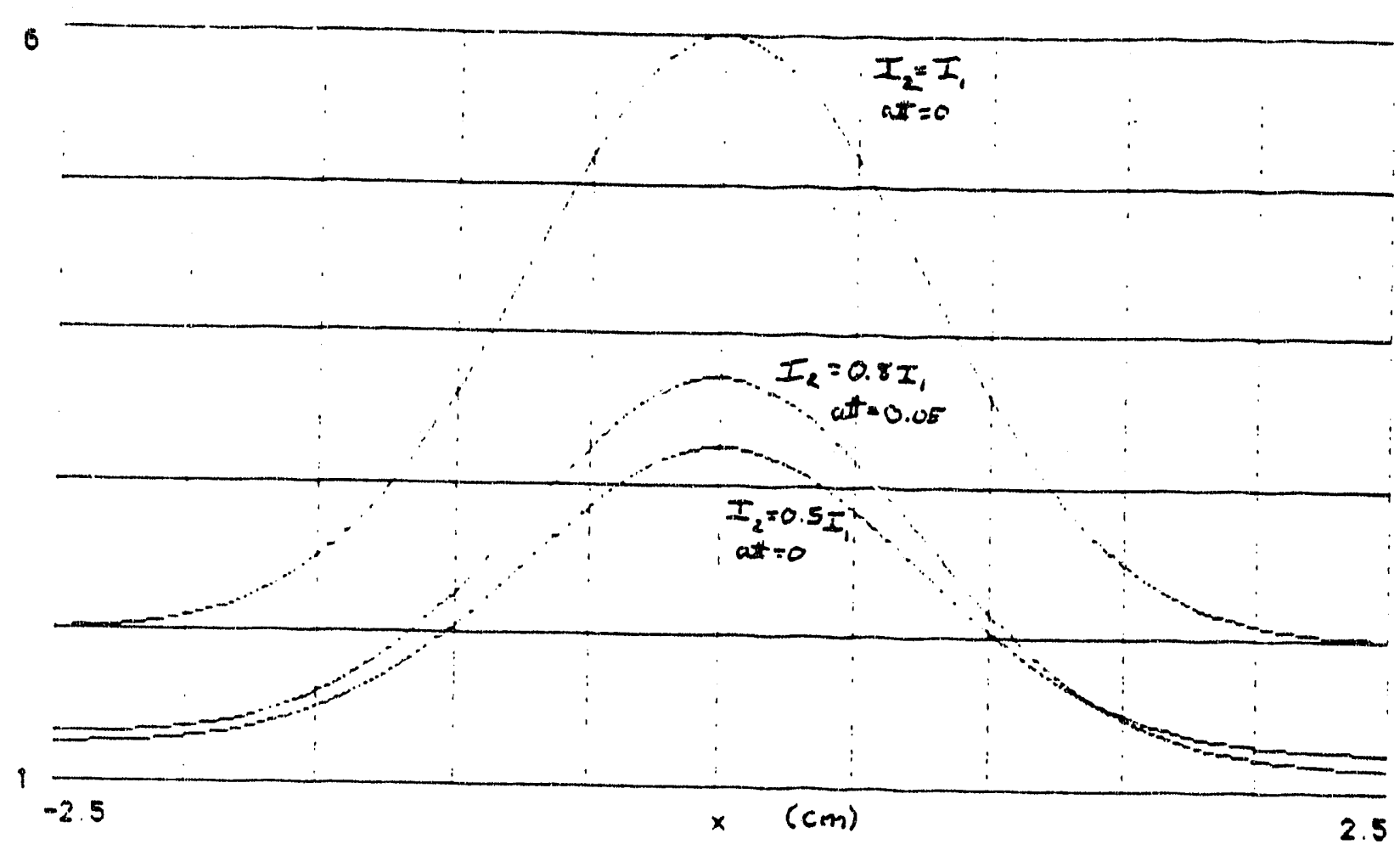

Figure 2-4: The fluorescence signal, $S(\tau)$, assuming a Gaussian pulse shape. The $y$-axis is normalized to $I_{1}$. The $x$-axis is in centimeters from the center of the cell. For the largest curve, $I_{2}=I_{1}$ and there is not attenuation through the dye. For the second curve $I_{2}$ is $80 \%$ of $I_{1}$ and the total attenuation through the cell is $5 \%$. For the third curve $I_{2}$ is half of $I_{1}$, and there is no attenuation. 


\subsection{Gaussian Beam Model}

If the laser is focused tightly in the dye cell, the plane wave model of the last section is no longer valid because the beam diameters will change significantly as the pulses traverse the dye cell. We can then model the laser output as a Gaussian beam which is a much more accurate representation of the real laser output. Thus, we have two Gaussian pulses traveling through each other in the dye. If the confocal parameter of the beam, $b=\frac{\pi w^{2}}{\lambda}$, is not much shorter than the length of the dye cell, we can treat the Gaussian beam as having a plane wave front, and we can use equations 2.14 and 2.27 . If the confocal parameter is too short equation 2.26 must be solved exactly.

Gaussian beams can be described by the slowly varying part of the vector potential. $\vec{A}[5 !$. It is more convenient to describe Gaussian beams in terms of the vector potential because the electric field is a complicated expression due to the curved phase fronts of Gaussian beams.

$$
\vec{A}=\dot{x} u(x, y, z) e^{i k z}
$$

For a Gaussian pulse,

$$
u=\frac{\sqrt{2}}{\sqrt{\pi} w} u_{\circ} A\left(t \pm \frac{n z}{c}\right) e^{-2 \frac{\left(z^{2}+y^{2}\right)}{w^{2}}} e^{-i \phi} e^{\frac{i t}{2 \pi}\left(x^{2}+w^{2}\right)}
$$

$w$ and $R$ are defined by

$$
w^{2}=w_{\circ}^{2}\left[1+\left(\frac{\lambda z}{\pi w_{0}^{2}}\right)^{2}\right]
$$

and

$$
\frac{1}{R}=\frac{z}{z^{2}+\left(\pi w_{0}^{2} / \lambda\right)^{2}}
$$

$w$ is a measure of the beam radius and describes the divergence of the beam; $w_{0}$ is the smallest beam radius. $R$ describes the curvature of the phase fronts of the beam.

The intensity is written in terms of $u$ as 


$$
I=\frac{1}{2} n \epsilon_{o} c \omega^{2}|u|^{2}
$$

Assuming that the two pulses are focused identically, we have,

$$
I_{\omega}=\frac{2}{\pi w^{2}} e^{-2 \frac{\left(\pi^{2}+y^{2}\right)}{w^{2}}}\left(P_{1}+P_{2}+2 \sqrt{P_{1} P_{2}} \cos \delta\right)
$$

where

$$
P_{1}=\frac{1}{2} n \epsilon c \omega^{2} u_{01}^{2} A^{2}\left(t \pm \frac{n z}{c}\right)
$$

is the power in the first pulse. $P_{2}$ is similarly defined and is the power in the isecond pulse. $\cos \delta$ is a rapidly varying - on the order of $\lambda$ - function of $z$. Uning equations $2.14,2.22$ and 2.24 , the recorded signal is

$$
S(\tau)=\iiint d x d y d t P_{f}=\frac{\alpha}{w^{2}} \int_{-\infty}^{+\infty} d t\left[P_{1}^{2}+P_{2}^{2}+4 P_{1} P_{2}\right]
$$

If the beam diverges significantly over the pulse length, it will not be possible to detect the full autocorrelation signal because there will not be enough fluorescience over the whole length. If an accurate autocorrelation signal is recorded, equation 2.37 can be used to correct for the effects of beam divergence on the signal. The width of the autocorrelation signal will be reduced by the decrease in fluorescence due to beam divergence.

\subsection{Copropagating Pulses}

If a pulse is split and then recombined after one half has been subjected to a variable delay, and then focused into a dye cell, the amount of fluorescence in the dye cell will be, as a function of the delay time, the autocorrelation of the pulse. This setup, shown in figure 2-5, is similar to SHG autocorrelator designs [6]. I will develop the equations to show that fluorescence power gives the autocorrelation signal. Assuming the pulses to be plane waves, 


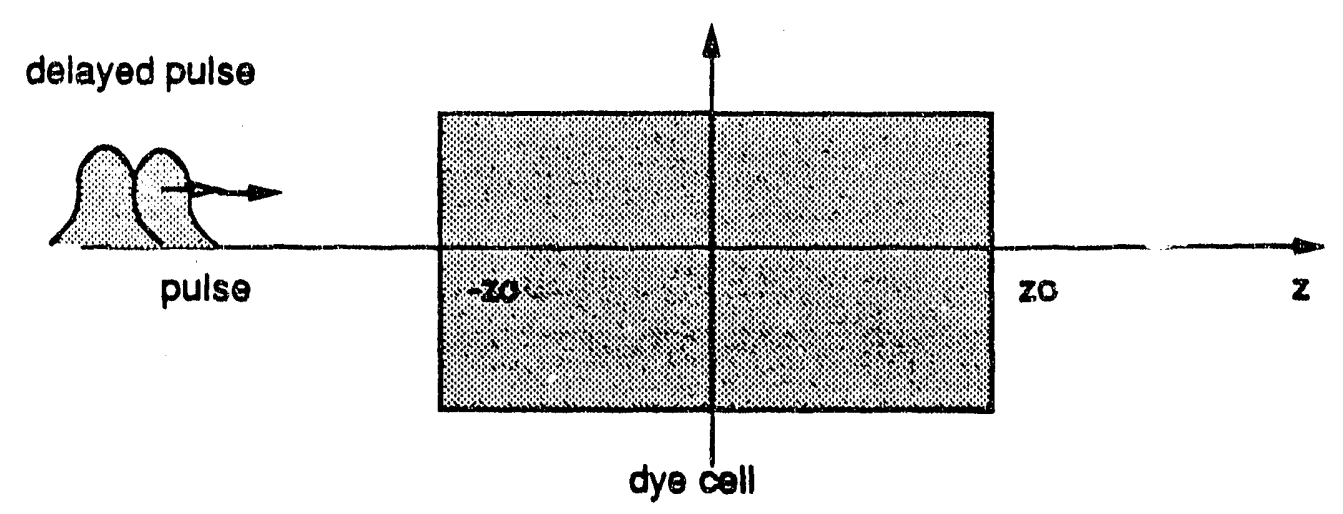

Figure 2-5: Co-propagating pulse autocorrelation

$$
E=E_{1} A\left(t-\frac{n z}{c}\right) e^{i(k z-\omega t)}+E_{2} A\left(t-\frac{n z}{c}+\delta t\right) e^{i(k z-\omega t+\omega \delta t)}+\text { c.c. }
$$

Using Equation 2.18,

$$
I_{\omega}=I_{1}\left(t-\frac{n z}{c}\right)+I_{2}\left(t-\frac{n z}{c}+\delta t\right)+2 \sqrt{I_{1}\left(t-\frac{n z}{c}\right) I_{2}\left(t-\frac{n z}{c}+\delta t\right)} \cos \omega \delta t,
$$

where $I_{1}$ and $I_{2}$ are defined as before. Substituting into equation 2.14,

$$
P_{f}=\alpha\left(I_{1}^{2}\left(t-\frac{n z}{c}\right)+I_{2}^{2}\left(t-\frac{n z}{c}+\delta t\right)+4 I_{1}\left(t-\frac{n z}{c}\right) I_{2}\left(t-\frac{n z}{c}+\delta t\right)\right)
$$

This is the same as equation 2.22 except that the overlap of the pulses is a function of the delay between the pulses, $\delta t$, and not distance. Thus, the autocorrelation of the pulses can be mapped out by changing the delay and measuring the fluorescence in the dye. 


\subsection{The Autocorrelation Signal}

Because we expect a certain output from the laser, we can calculate what the expected autocorrelation signal should be. The laser is actively modelocked, and, thus, the steady state output will be a solution to the Mathieu equation [5]. Under the assumptions of well separated pulses in a cavity tuned to the modelocking frequency, the output pulse shape will be Gaussian [5]. We thus expect, from equation 2.25, the autocorrelation signal to be

$$
G(\tau) \propto \int_{-\infty}^{+\infty} e^{-\omega_{p}^{2} t^{2}} e^{-\omega_{p}^{2}(t-\tau)^{2}} d t \propto e^{-\frac{\omega_{p}^{2}}{3} \tau^{2}}
$$

where $\omega_{p}$ is a parameter dependent upon the laser characteristics. Thus if we define the pulse width, $\Delta t$, to be $\frac{1}{\sqrt{2} \omega_{p}}$, the autocorrelation width is $\sqrt{2} \Delta t$, mapped onto space by the relation, $z=\frac{s}{n} t$. This autocorrelation signal will be superimposed on a background as shown in figure $2-4$.

It is important to know what sort of autocorrelation signal to expect so that the results can be correctly interpreted. For coherent laser pulses the contrest of the autocorrelation signal, $S(0) / S(\infty)$, will be 3 to 1 as is shown in figure 2-4. The autocorrelation signal for a non-modelocked laser will resemble figure 2-6 [6]. There is a spike at $\tau=0$ because even a noisy signal is in phase with itself. The width of the spike corresponds to the coherence time of the laser. The smaller the linewidth of the laser, the larger the width of the spike. Thus, for noise, the contrast ratio will be $S(0) / S(\infty)=3 / 2$.

The autocorrelation signal for burst of noise is shown in figure 2-7. The spike at $\tau=0$ is due to the coherence of the noise as in figure 2-6. The wider structure is due to the width and structure of the burst.

\subsection{Imperfect Beam Overlap}

I have included the possibility that the two pulses are of unequal intensities in the equations thus far, but I have implicitly assumed that there was perfect beam overlap 


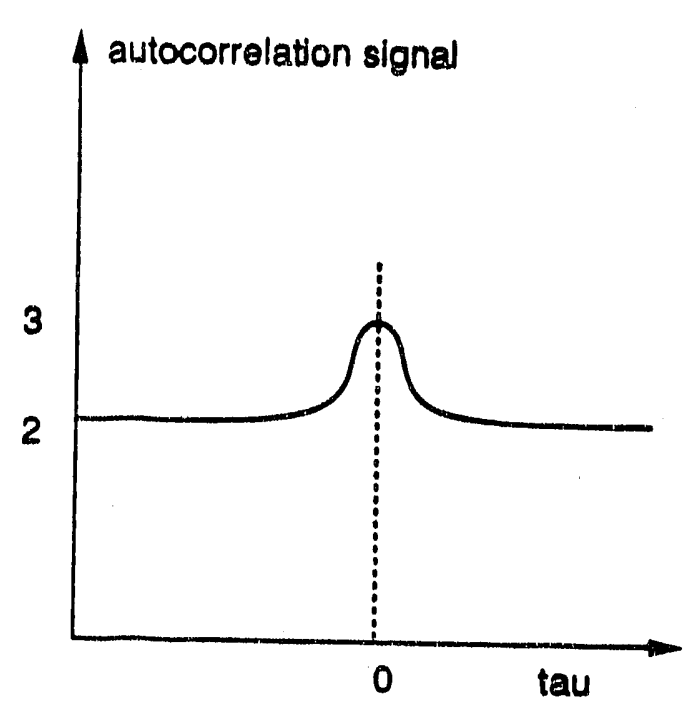

Figure 2-6: The autocorrelation signal of a non-modelocked laser

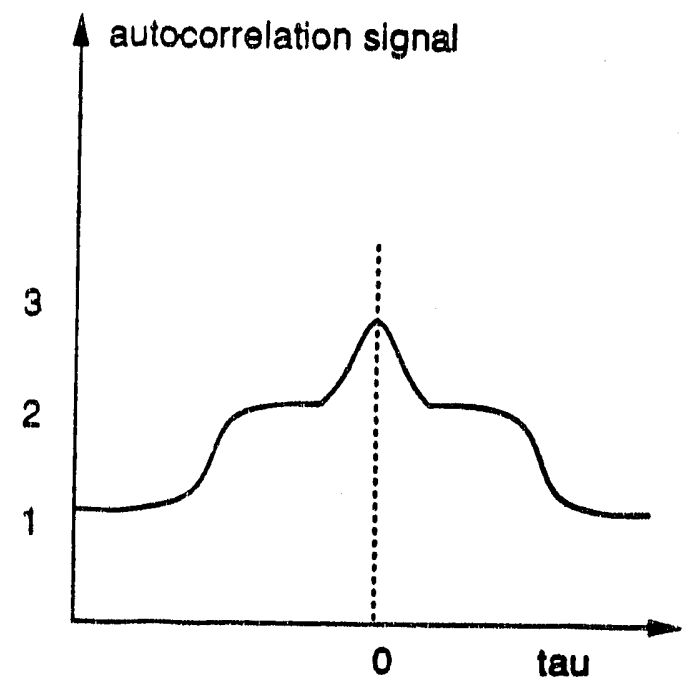

Figure 2-7: The autocorrelation signal of a burst of noise noise 
-i.e. that the bearns passed through each other perfectly. Although the effects of imperfect beam overlap are included in equation 2.24 , the analysis up to this point has assumed that the heams were perfectly overlapped so that the integrals over $x$ and $y$ in equation 2.25 cancel out. But this might not be the case. In fact, a system might be designed with imperfect beam overlap to prevent the beams from returning along the same paths and feeding back in to the laser. Also, in practice it is difficult to align the beams perfectly [9].

The effect of imperfect beam overlap will be to reduce the probability for twophoton absorption with one photon from each pulse which is the reduction of the autocorrelation signal. Thus, the autocorrelation signal will be reduced and the contrast ratio will also be reduced. We can quantify the effect of imperfect beam overlap by considering the reduction in the autocorrelation signal. I will assume that the pulse shape is Gaussian.

When the beams are perfectly overlapped the spatial integration of the autocorrelation function will be

$$
G\left(\tau, x_{0}=0, y_{0}=0\right)=\frac{\int d x e^{-x^{2} / w^{2}} e^{-x^{2} / w^{2}} \int d y e^{-y^{2} / w^{2}} e^{-y^{2} / w^{2}} \int I_{1}(t) I_{2}(t-\tau) d t}{\int d x e^{-2 x^{2} / w^{2}} \int d y e^{-2 y^{2} / w^{2}} \int d t I_{1}^{2}+\int d x e^{-2 x^{2} / w^{2}} \int d y e^{-2 y^{2} / w^{2}} \int d t I_{2}^{2}} .
$$

When the beam centers are separated by $\rho_{0}=\sqrt{x_{0}^{2}+y_{0}^{2}}$, the spatial integration of the autocorrelation function will be

$$
G\left(\tau, x_{0}, y_{0}\right)=\frac{\int d x e^{-x^{2} / w^{2}} e^{-\left(x-x_{0}\right)^{2} / w^{2}} \int d y e^{-y^{2} / w^{2}} e^{-\left(y-y_{0}\right)^{2} / w^{2}} \int I_{1}(t) I_{2}(t-\tau) d t}{\int d x e^{-2 x^{2} / w^{2}} \int d y e^{-2 y^{2} / w^{2}} \int d t I_{1}^{2}+\int d x e^{-2\left(x-x_{0}\right)^{2} / w^{2}} \int a y e^{-2\left(y-y_{0}\right)^{2} / w^{2}} \int d t I_{2}^{2}} .
$$

The ratio of equations 2.42 and 2.43 will indicate the reduction in the contrast ratio. The denominators are equal, so the ratio is

$$
\frac{\int d x e^{-x^{2} / w^{2}} e^{-\left(x-x_{0}\right)^{2} / w^{2}} \int d y e^{-y^{2} / w^{2}} e^{-\left(y-y_{0}\right)^{2} / w^{2}}}{\int d x e^{-x^{2} / w^{2}} e^{-x^{2} / w^{2}} \int d y e^{-y^{2} / w^{2}} e^{-y^{2} / w^{2}}}=e^{-\left(x_{0}^{2}+y_{0}^{2}\right) / 2 w^{2}} .
$$

Equation 2.44 measures the effects of beam misalignment. It is used in the next 
chapter to evaluate the consequences of misaligning the beams for the purpose of irolation.

\subsection{Summary}

The important design considerations can be found in the mathematical treatment of the theory given in this chapter. In order to generate enough fiuorescence, the IR beam must be intense. But the confocal parameter of the beam must not be too short or the whole nutocorrelation signal will not be seen. Thus, there is an important trade-off between generating high intensity and beam divergence. Figure 2.4 shows that the width of the autocosrelation signal is not severely affected by attenuation and unequal beam intensitics, although they reduce the contrast ratio and skew $S(r)$; so the pulse width can still be inferred from the signal. The last section indicates the importance of aligning the beams so that they overlap. 


\section{Chapter 3}

\section{Design}

In this chapter, I will describe different autocorrelator designs and their relative advantages and disadvantages.

Figure 3.1 is a design in which the pulse from the laser is split with a 50 percent beam splitter and autocorrelated with itself in the dye. This is the standard two. photon fluorescence autocorrelator design [9].

Figure 3.2 is a design in which the pulse is reflected back on itself in the dye cell so that the first half of the pulse is correlated with the second half. This design is nice and compact but if the beam is focused down into the dye cell care must be taken not to damage the mirror. Only half the autocorrelation pulse is seen, but this should not matter because the autocorrelation function is symmetric, although having the whole autocorrelation signal would yield more accurate results.

Figure 3.3 is a design in which each pulse is not autocorrelated with itself, but instead is correlated with the next pulse in the train. Because two pulses are being used the signal to noise ratio of this scheme should be higher than that of schemes in which one pulse is split and autocorrelated with itself. It also has the advantage that the beam is not focused near any of the optics so that the possibility of damaging the optics is minimized when high energy pulses are used.

An important consideration is alignment. Alignment is critical because if the beams are misaligned by even thousandths of a centimeter the autocorrelation signal can become significantly smaller. Thus it is important that there be an effective 


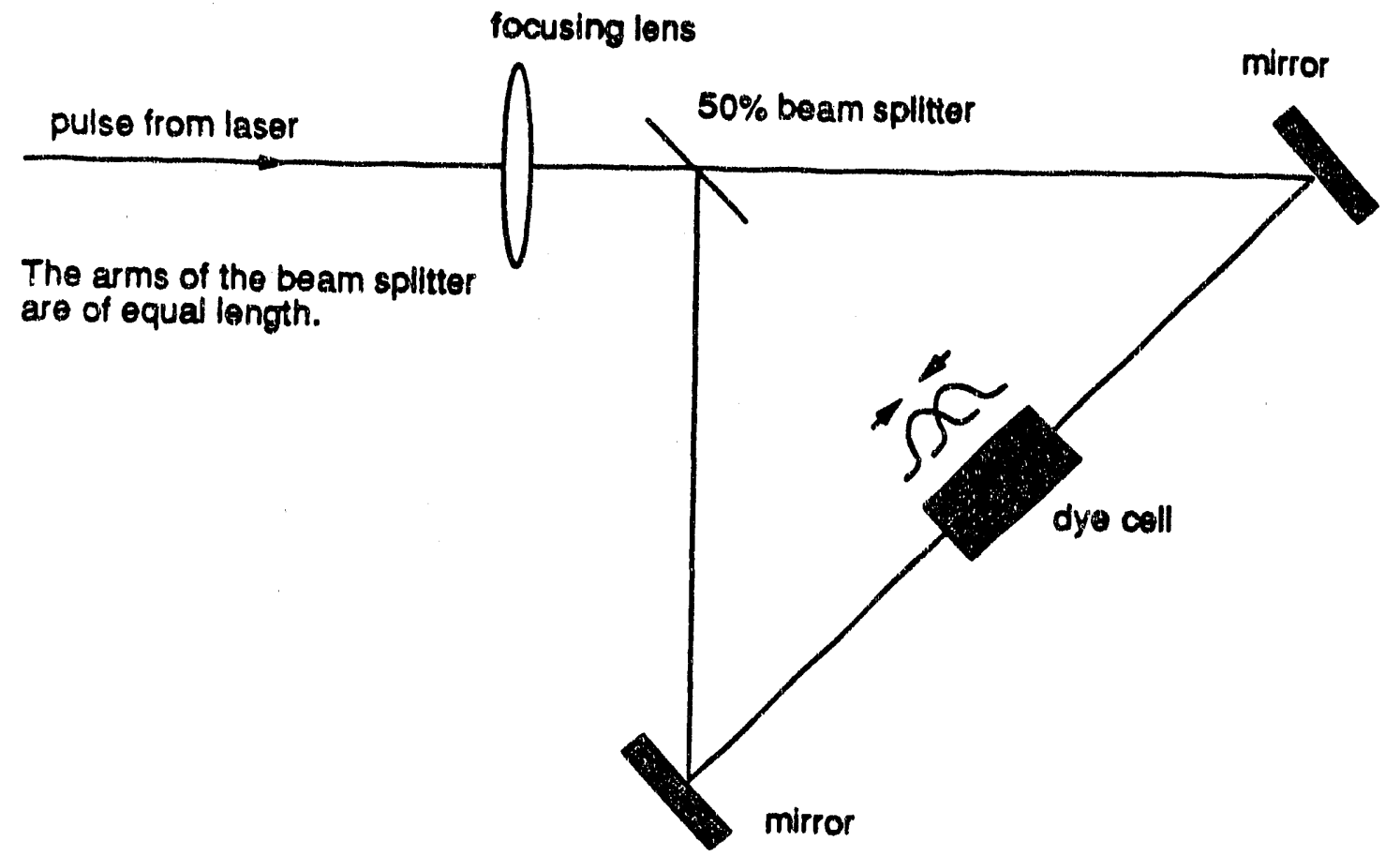

Figure 3-1: 50 percent beam splitter design

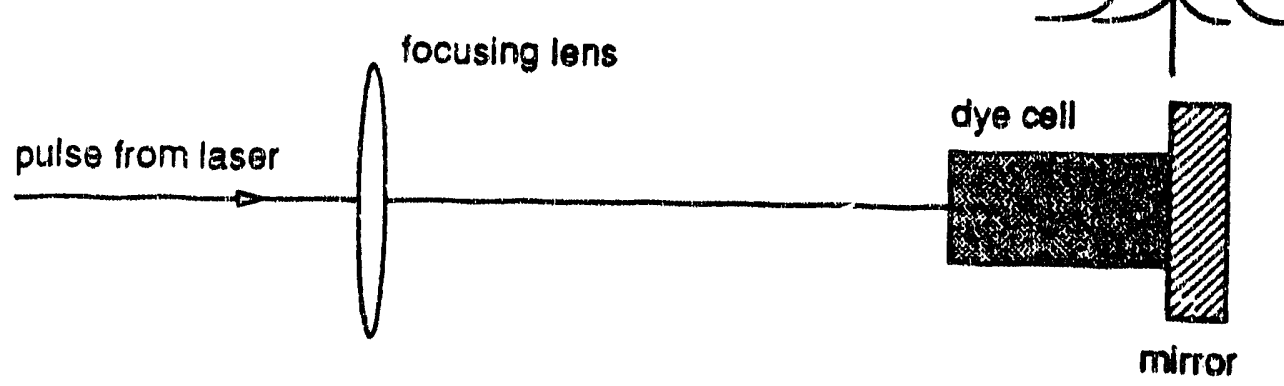

Figure 3-2: The pulse is reffected back on itself in the dye so that the beginning of the pulse is autocorrelated with the end 


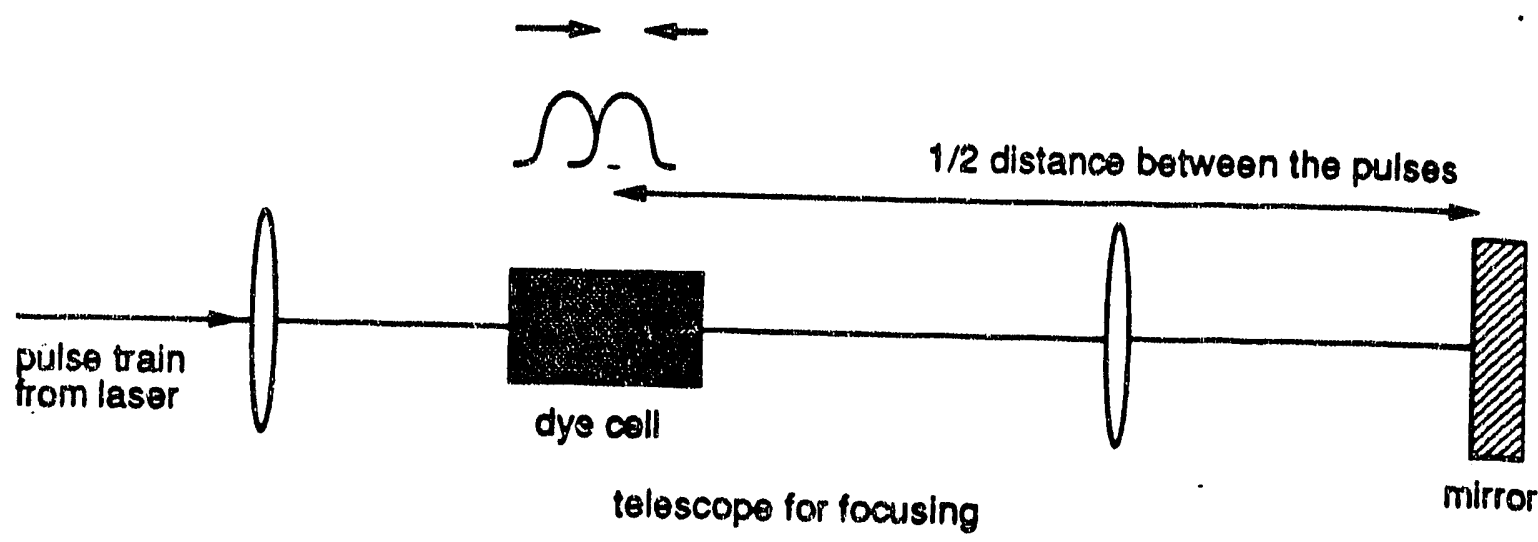

Figure 3-3: Each pulse is autocorrelated with the next pulse in the train.

method for aligning the system. It is preferable that the system be aligned with a Helium-Neon laser to avoid wearing out the laser flash lamp. Once the system is aligned to the Helium-Neon laser, two pinholes aligned to the Helium.Neon laser can be used to align the ND:YLF.

The 50 percent beam splitter in the first design mentioned above has a port which is not followed by any optics. This port is helpful in alignment because both beams going through the dye cell will then be partially reflected or transmitted through this port so that the system can be aligned by adjusting until both the beams are aligned going through this port. The advantage is that the beams can be looked at a long distance away where the distance between the two beams will be very sensitive to the alignment of the beams going through the dye cell; when theses two beams overlap perfectly the system is aligned. The other two designs do not have an extra port as in the first design so that alignment is more difficult. But the two beams refiected back towards the laser can be looked at as far away as possible and adjusted so that they overlap.

\subsection{Isolation}

One significant difficulty with all the above designs is that the light pulses travel back along the same paths so that the the laser power is coupled back into the laser where it has the potential for damaging the laser components. This problem arises when 
the pulses from the laser oscillator are amplified by an additional rod and flash lamp outside of the oscillator cavity. Then any light reflected back is amplified again in the external rod and the pulses coupled back into the oscillator are much more intense than the original pulses. Thus, a limit is put on how much power can be used in these designs.

There are two ways to reduce or eliminate the amount of light that is coupled back in to the laser. The simplest and most effective is to have the returning beam simply not go back into the laser. This requires, for the above designs, that the beam be misaligned. Whether or not the beam can be misaligned enough to prevent feedback without seriously affecting the performance of the autocorrelator must be evaluated.

The other method is to insert an isolator. An isolator is a device which does not pass the returning beam. They operate by rotating the polarization so that the returning beam is polarized orthogonally to the incoming beam and can be eliminated with a polarizer. Figure $3-4$ is a diagram of the isolator design I used.

The isolator consists of a polarizer aligned with the polarization of the laser and a quarter wave plate with its axis at $45^{\circ}$ to the electric field so that the beam coming out of the quarter wave plate is ircularly polarized. If this beam is reflected by a mirror it will return through the quarter wave plate and come out orthogonally polarized to the incoming beam (as if it went through a half wave plate) so that it will not be passed by the polarizer.

The isolation is not perfect because the polarizer does not absorb all the reflected light and the quarter wave plate does not work jdeally on a beam with a curved phase front and because of other small effects. I measured the extinction ratio - the ratio of the amount of light returned to the amount of light going in - to be 0.01. A difficulty with this scheme is that most bearnsplitters ate polarization sensitive, and the isolator puts out circularly polarized light so that it cannot be used with the 50 percent beamsplitter design.

Now I will consider the effect of misaligning the beams. The dye cell is $5 \mathrm{~cm}$ long, so suppose that we decide to misalign the beam so that the centers of the two pulses are $u_{0}$ away from each other at the end of the dye cell. Referring to equation 2.44 , 
Polarizer axis is vertical.
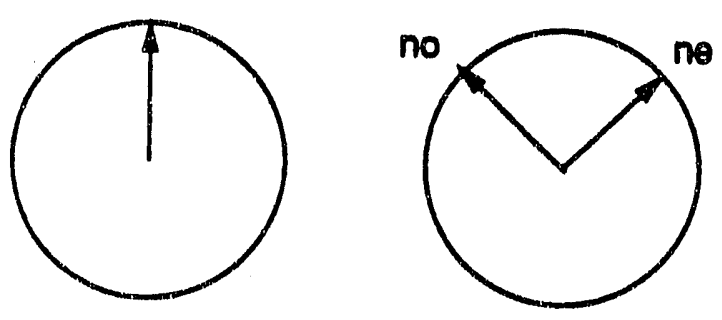

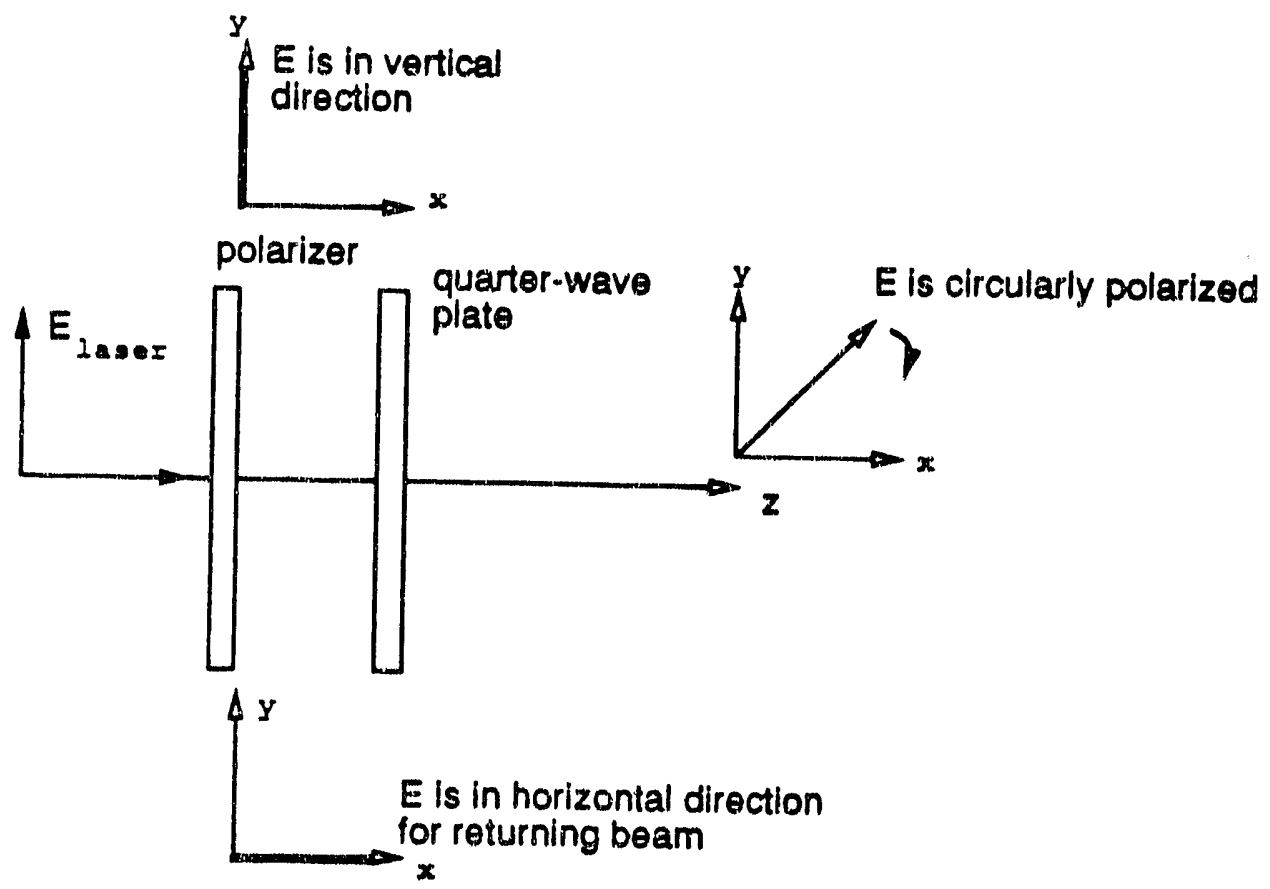

Figure 3-4: Optical Isolator 


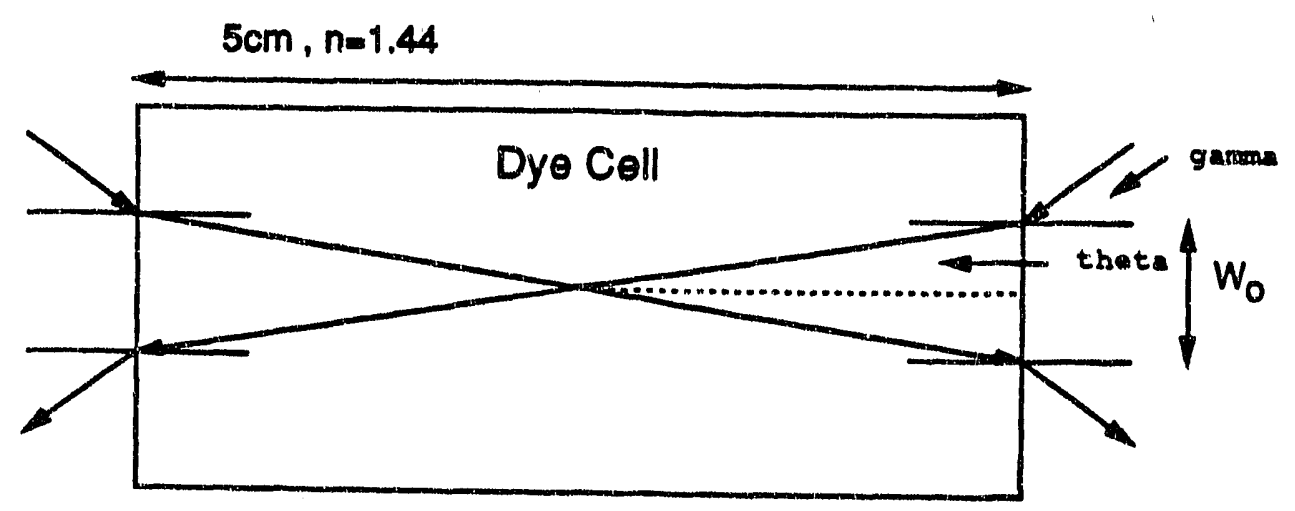

Figure 3-5: Isolation by beam misalignment

this will reduce the autocorrelation signal by about 60 percent at the edge of the dye cell. Will this misalign the beam enough to provide significant isolation?

Consider the 50 percent beam splitter design. Figure $3-5$ shows the important parameters. We are interested in the values of the beam waist and the distance from the dye cell such that the two beams are separated. A distance 1 from the dye cell the centers of the two beams are separated by a distance,

$$
d \cong 2 l\left(1.44 \frac{w_{\circ}}{5 \mathrm{~cm}}\right)+w_{\circ}
$$

and the beam radius is

$$
w^{2} \cong w_{0}^{2}\left(1+\left(\frac{\lambda l}{\pi w_{0}^{2}}\right)^{2}\right)
$$

The statement that the two bearns are separated is

$$
d>2 w
$$

For example, for the beams to separated $2 \mathrm{~cm}$ away from the cell, the beam waist must be greater than $130 \mu \mathrm{m}$; For the beams to be separated $25 \mathrm{~cm}$ away, the waist must be greater than $104 \mu \mathrm{m}$.

The main problem with this technique is that it makes the problem of interpreting the autrcorrelation signal more difficult because one must account for the fact that the autocorrelation signal is dependent on the distance from the point of intersection 
of the two beams. It is also undesirable because we would like as much autocorrelation signal as possible.

Another possibility is to have the two beams focus at different points so that the beam that returns to the lens will diverge. This will happen if the beam waists are closer to the lens than the focal distance. The problem with this scheme is that a reasonable shift in the focus distance will not provide significant isolation. For the 50 percent beamsplitter design, using a lens with a $40 \mathrm{~cm}$ focal length, I calculated the effect of separating the focal points of the two beams by $1 \mathrm{~cm}$. Assuming that the laser is $50 \mathrm{~cm}$ away from the lens, the beam radius would only be $2.55 \mathrm{~mm}$ for an initial beam radius of $2 \mathrm{~mm}$, the output radius of the ND:YLF beam.

\subsection{Copropagating Pulse Design}

The 50 percent beamsplitter design is the traditional TPF autocorrelator design, and the other two designs work on the same principle. The detection schemes used for these designs typically use film or video cameras to image the fluorescence [3] [4] [9].

Autocorrelators that use second harmonic generation (SHG) use Michelson Interferometers with an adjustable arm length to adjust the delay between the pulses [6]. As I showed in chapter 2, TPF can also be used to measure the autocorrelation of copropagating pulses by varying the delay of one of the pulses. As in SHG autocorrelators, this can be done with a Michelson interferometer as shown in figure 3-6.

The advantage of this scheme is that the beam can be focused as tightly as possible in the dye cell to create more fluorescence and that the whole signal can be measured by one large area detector and doesn't have to be imaged. As sensitive a detection scheme is not needed. The quenching and bleaching of the dye are the constraints on how tightly focused the beam can be in the dye. The disadvantage of this scheme is that the autocorrelation signal cannot be acquired in a single shot. 
l.

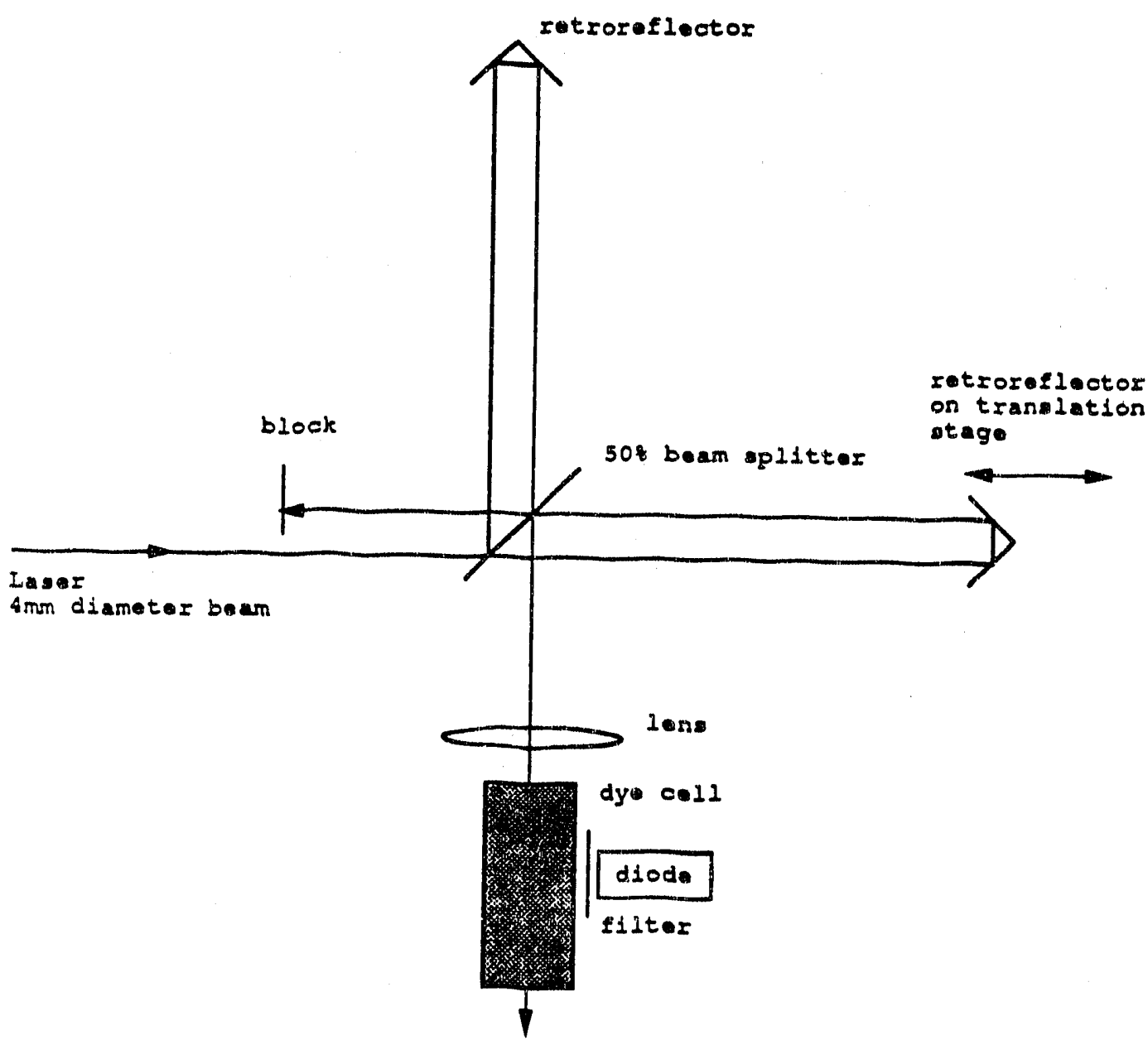

Figure 3-6: Autocorrelator using TPF and a Michelson Interferometer 


\section{Chapter 4}

\section{Imaging and Detection}

Without the preamplifier the laser produces $\sim 0.5 \mathrm{~mJ}$ per shot, and each shot contains about 100 pulses. With the preamplifier at a gain of 10 , the energy in each shot is about $4 \mathrm{~mJ}$. In this section I will calculate how much laser energy is necessary to obtain a clear signal if we image the signal onto a diode array and if we focus the signal onto a single large-area photodiode. For the copropagating pulse design the signal would be focused onto a single photodiode. For the designs mentioned at the beginning of the last chapter, the autocorrelation signal is collected in one shot as a function of distance and needs to be imaged onto a diode array or other recording device such as film or a CCD camera.

\subsection{Imaging Optics}

To image the signal onto a $1 \mathrm{~cm}$ diode array we need to demagnify it by a factor of five, and it is important to do this as efficiently as possible. The problem is that the fluorescence signal - the autocorrelation signal - is radiated isotropically and enough of it must be collected by the imaging optics so that the signal can be detected. An imaging scheme using two lenses is proposed because it is more efficient than a simple one lens design [10]. It is more efficient than a one lens scheme because, for lenses of the same focal length, the first lens, in the two lens scheme, will be closer to the signal and, thus, more of the signal will be collected by the lens. 


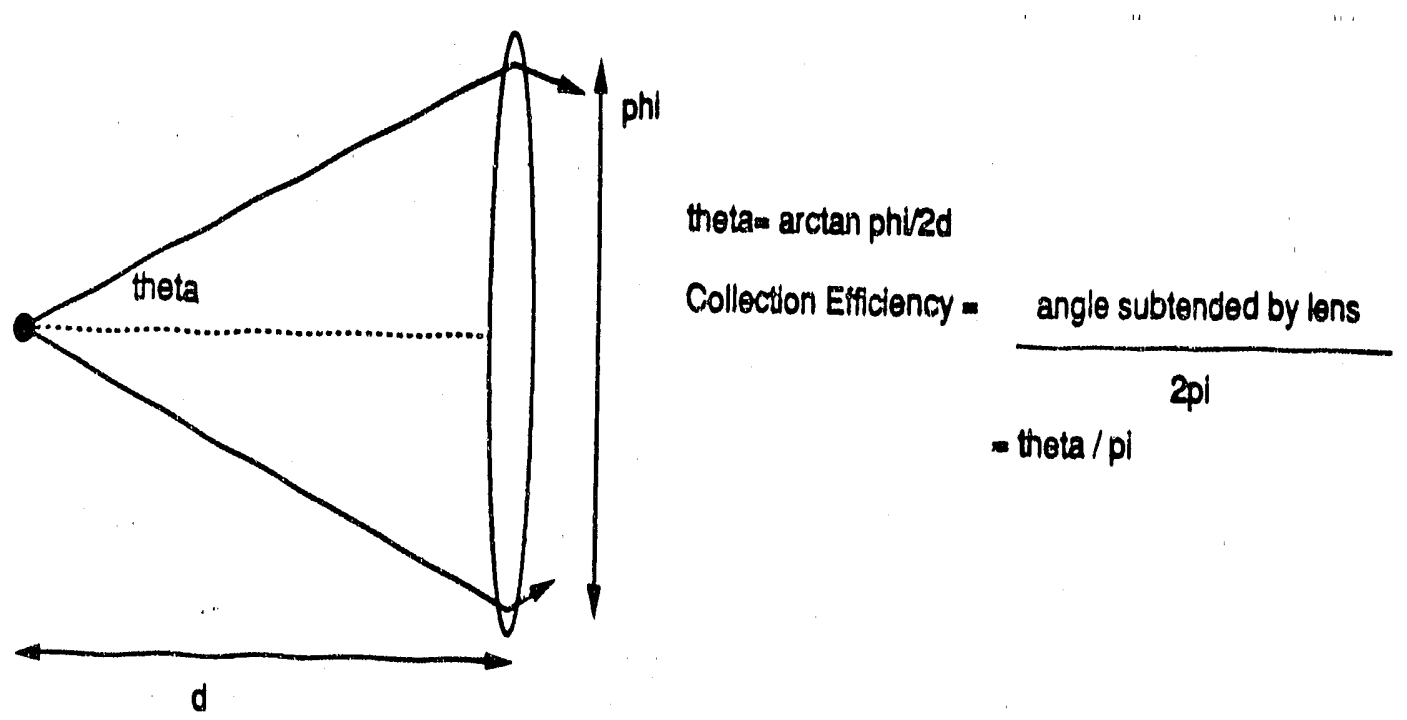

Figure 4-1: Calculating the collection efficiency of imaging optics

The design of the imaging optics is calculated using the ray optics equation for the image created by a lens,

$$
\frac{1}{s_{0}}+\frac{1}{s_{i}}=\frac{1}{f}
$$

where $s_{0}$ is the distance of the object from the lens and $s_{i}$ is the distance of the image from the lens in the opposite direction. The magnification is $M=\frac{s_{i}}{\omega_{0}}$. Thus, for five times demagnification using a single lens, $s_{0}=6 f$ and $s_{i}=6 f / 5$. Because the fluorescence is along a $5 \mathrm{~cm}$ line, for the purpose of calculation, I will assume that all the fluorescence is radiated out with cylindrical symmetry. To calculate how much light is captured by the lens, the angle subtended by the lens is calculated, as in figure 4-1. The collection efficiency for one lens is

$$
\nu_{\mathrm{c}}=\frac{1}{\pi} \arctan \frac{\phi}{12 f} \approx \frac{\phi}{12 \pi f},
$$

where $\phi$ is the lens diameter. This is the collection efficiency near the center of the lens. The collection efficiency will fall off at the ends of the lens because the lens is circular. The ratio of lens diameter to focal length is important. Using a glass, biconvex 2inch Newport lens, the distance from the fluorescence streak to the lens is $22.86 \mathrm{~cm}$ and the collection efficiency is 0.031 . ( $\phi$ is $4.45 \mathrm{~cm}$ because this is the usable diameter when the lens is mounted.) 
pixel height

pixel width

saturation

sensitivity
$250 \mu \mathrm{m}$

$15 \mu \mathrm{m}$

$0.18 \mu \mathrm{J} / \mathrm{cm}^{2}$ (output $4 \mathrm{pC}$ ) (at $760 \mathrm{~nm}$ )

$25 \mathrm{pA} /\left(\mu \mathrm{W} / \mathrm{cm}^{2}\right)$ (at $\left.760 \mathrm{~nm}\right)$

Table 4.1: K series EG\&G Reticon diode array (data from the 1989 Image Sensing Products Databook)

\subsection{Imaging Detection}

We would like to image the autocorrelation signal in the $5 \mathrm{~cm}$ dye cell onto a $1 \mathrm{~cm}$ diode array. Table 4.1 lists the important characteristics of the diode array which is a $\mathrm{K}$ series array manufactured by EG\&G Reticon. The array is controlled by an RC100 board which outputs a 2.5 Volt signal at saturation.

I will calculate how much nominal power is required to saturate the pixels of the array. The calculation is approximate, and the expectation is that the power calculated will provide a measurable signal; it is unlikely that the diode array will be saturated. $0.18 \mu \mathrm{J} / \mathrm{cm}^{2}$ is needed to saturate a pixel at the optimum detection wavelength. The efficiency at $575 \mathrm{~nm}$ is 0.8 that at $760 \mathrm{~nm}$, the optimum wavelength. Thus, $0.225 \mu \mathrm{J} / \mathrm{cm}^{2}$ of fluorescence will saturate the pixel. Each pixel has an area of $3.75 \times 10^{-5} \mathrm{~cm}^{2}$, so that $8.4 \times 10^{-6} \mu \mathrm{J}$ will saturate a pixel. The energy from a pulse captured by a pixel is

$$
E_{p}=T_{f}\left(\alpha I^{2}\right) V_{p} r_{p}
$$

where $T_{f}$ is the transmission of filter, $V_{p}$ is the volume of illuminated dye that reaches pixel, and $\tau$ is the time duration.

We would like to calculate what intensity, $I$, is required for $E_{\mathrm{p}}$ to be $8.4 \times 10^{-6} \mu \mathrm{J}$. An Oriel broadband interference filter centered at $600 \mathrm{~nm}$, will transmit a minimum of 50 percent of the light at $575 \mathrm{~nm}$. Using a $0.01 \mathrm{M}$ solution of Rhodamine $6 \mathrm{G}, \alpha$, defined in equation 2.15 , is $2.275 \times 10^{-12} \mathrm{~cm} / \mathrm{W}$. The volume of illuminated dye is approximately a cylinder of radius $w_{0}$ the length of the dye cell. The amount of this 
fluorescence that reaches a pixel will be the amount captured by the lens in the vertical direction - perpendicular to the fluorescence streak. Along the fluorescence streak, a length of 125 um will reach a particular pixel because the streak is demagnified five times.

$$
V_{p}=\frac{1}{\pi} \arctan \left(\frac{\phi}{2 s}\right) \pi w_{o}^{2}\left(5 l_{p}\right)=6.17 \times 10^{-8} \mathrm{~cm}^{3}
$$

This value is calculated for $w_{0}=67 \mu \mathrm{m}$, the beam waist if the $2 \mathrm{~mm}$ radius laser output is focused with a $40 \mathrm{~cm}$ focal length lens.

The nominal pulse length is 100 ps and there are approximately 100 pulses in each shot, so I will take $\tau$ to be a $100 \times 100$ ps. The intensity needed to saturate the pixels is then calculated to be $110 \mathrm{MW} / \mathrm{cm}^{2}$. Each shot contains 100 pulses so this intensity corresponds to $0.2 \mathrm{~mJ} /$ shot.

According to this calculation we should expect to see fluorescence, but, as stated before, this calculation makes many assumptions. $0.225 \mu \mathrm{J} / \mathrm{cm}^{2}$ is the saturation exposure for the integration time, $1.92 \mathrm{~ms}$, of the array which is the period of the scan of the array and, consequently, corresponds to the time between each measurement of the diode charge. Between measurements the diode charge is stored on a capacitor. The length of the $Q$-switched shot is $\sim 750 \mathrm{~ns}$ which is much less than the integration time. The EG\&G Reticon diode array specifications do not provide information on the configuration and time constants of the diode circuits.

The calculation assumes that all the laser energy is in the pulses, but if the laser is not perfectly modelocked, a significant amount of energy could be outside the laser pulses [3]. Thus the intensity would be much less for the same energy per shot. When setting up and aligning the autocorrelator, the distribution of laser energy will be an additional uncertainty. If the predicted amount of fluorescence is not measured, it will be difficult to determine whether the laser or the alignment is the cause.

In the calculation I also assume for simplicity that all the fluorescence is radiated radially, ignoring what happens at the ends of the streak. Energy is also lost in reflections off the dye cell and lenses. By calculating what it takes to saturate the 


\begin{tabular}{cc|cc}
$\mathrm{J}$ & $\mathrm{K}$ & $\mathbf{Q}$ & not $Q$ \\
\hline $\mathrm{L}$ & $\mathrm{L}$ & $\mathrm{Q}_{0}$ & not $Q_{0}$ \\
$\mathrm{H}$ & $\mathrm{L}$ & $\mathrm{H}$ & $\mathrm{L}$ \\
$\mathrm{L}$ & $\mathrm{H}$ & $\mathrm{L}$ & $\mathrm{H}$ \\
$\mathbf{H}$ & $\mathbf{H}$ & toggle &
\end{tabular}

Table 4.2: Logic Table for a J.K tlip-tiop

array, I hope to ensure that the system is robust enough to writhstand unaccounted for losses, but it is not clear that this calculation provides an accurate estimate because of all the assumptions. Alignment of the array is also very critical because a narrow streak is being imaged on a narrow array. As will be seen, there were many difficulties recording the signal on the array.

\subsubsection{Reticon Triggering}

The Reticon Array system has internal clock and puts out a signal that is scan of the pixels linear in time so that the time from the start of the scan is proportional to the position along the array of the pixel whose voltage is displayed at that time. The repetition rate of the scan is not synchronous with the laser. If the oscilloscope is triggered off the laser, the position of the start of the scan on the oscilloscope will vary from shot to shot. A J.K flip-flop can be used to create a signal, synchronous with the array, that will irigger the oscilloscope every shot. Thus, the signal on the oscilloscope will correspond to the autocorrelation signal. Table 4.2 is the logic table for an edge-triggered J.K flip-flop.

The trigger signal from the laser and the start signal from the array, which signals the start of the scan, are combined to set and reset the flip-flop, creating a pulse that is synchronous with the array. Figure $4-3$ is the circuit diagram for the design. The AND gate combines the laser trigger and the start signal so that the flip-flop set occurs synchronously with the start signal when the laser trigger is high. The flip-fop is reset when the laser trigger goes low. The trigger delay in the oscilloscope (A Philips digital oscilloscope) can be used to center the fluorescence signal on the 


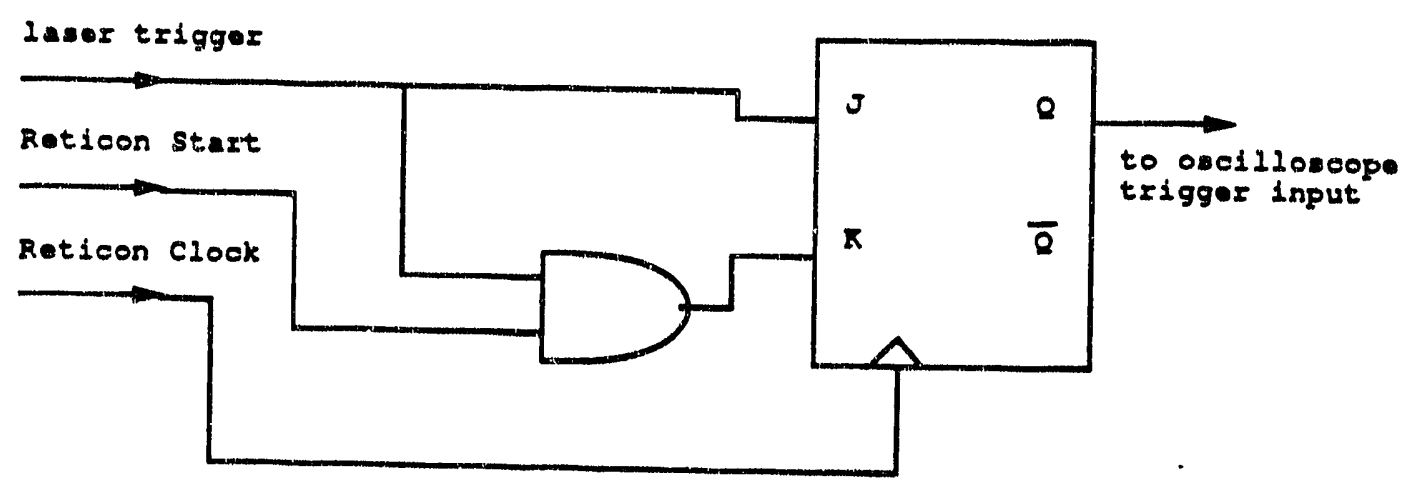

Figure 4-3: A circuit to create a trigger signal for the oscilloscope

$\begin{array}{lc}\text { diode area } & 100 \mathrm{~mm}^{2} \\ \text { diameter } & 0.444 \mathrm{in} \\ \text { sensitivity } & 0.45 \mathrm{~A} / \mathrm{W} \text { (at } 850 \mathrm{~nm} \text { ) }\end{array}$

Table 4.3: United Technologies PIN-10D diode characteristics

screen.

\subsection{Tutal Fluorescence Detection}

For the copropagating pulse design described in the last chapter, the fluorescence does not need to be imaged. All of it is collected onto one photodiode, and the photodiode signal is recorded as a function of the adjustable delay. Table 4.3 lists the relevant characteristics of the United Technologies PIN-10D diodes used.

Again, I will calculate what signal we can expect to see. Because this method of measuring the autocorrelation requires taking data over at least several puises, there is no question of using the amplifier. There will be $\sim 0.5 \mathrm{~mJ} /$ shot but the beam can be focused very tightly because the streak is not being imaged.

If the $2 \mathrm{~mm}$ radius $1.054 \mathrm{um}$ beam is focused using a $50 \mathrm{~mm}$ focal length lens, the focused beam will have a beam waist of $w_{0}=8.4 \mu \mathrm{m}$ and a confocal parameter $b=0.3 \mathrm{~mm}$. Because the beam will diverge rapidly, we can estimate the volume of illuminated dye to be $\pi w_{0}^{2} b=6.65 \times 10^{-5} \mathrm{~cm}^{3}$. The fluorescence energy generated by 


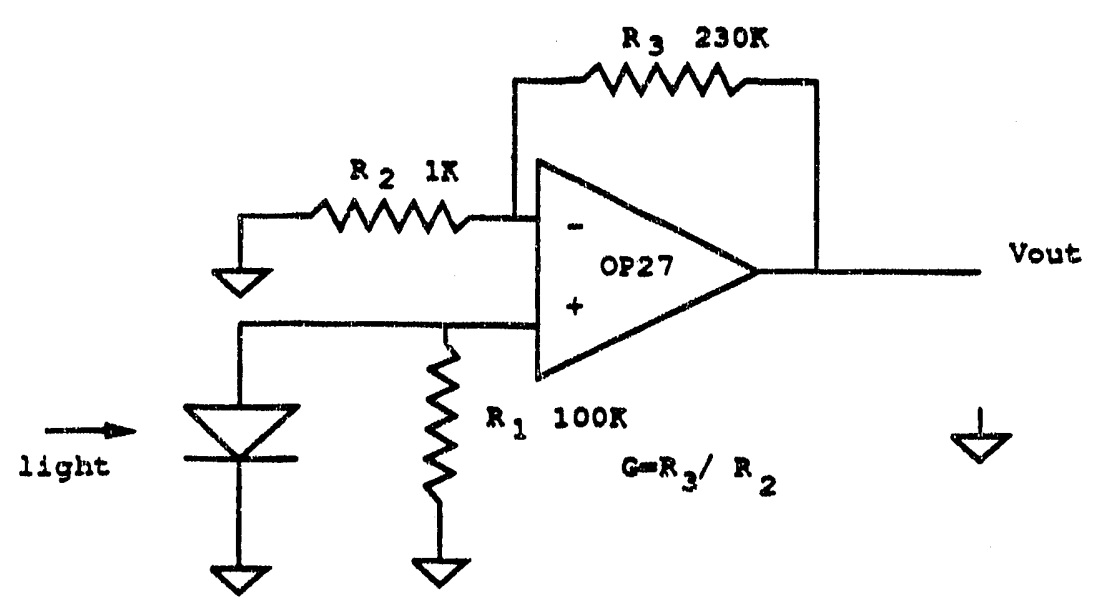

Figure 4-4: Photodiode Circuit

a pulse will be

$$
E_{f}=\alpha I^{2} V \tau=7.73 \times 10^{n-9} \mathrm{~J}
$$

Because the dye has a persistence on the order of $50 \mathrm{~ns}$ [4], the fluorescence energy will not be released in $100 \mathrm{ps}$. There is a pulse every 7.5ns, so the amount of energy created by one pulse will be, on average, released eve:y $7.5 \mathrm{~ns}$ so that the fluorescence power will be $1.03 \mathrm{~W}$. Because the confocal parameter is small compared to the diode size, I will assume that the fluorescence is radiated spherically. If the diode is simply placed close to the dye cell without any focusing optics the collection efficiency will be the fractional surface area of a sphere subtended by the diode area. The closest the diode can be brought to the dye cell is $3 \mathrm{~cm}$, for which the collection efficiency is 0.0086 . Accounting also for 50 percent loss in the filter, $4.43 \times 10^{-3} \mathrm{~W}$ will reach the diode. Scaling the sensitivity at $850 \mathrm{~nm}$ by the relative sensitivity, the sensitivity at $575 \mathrm{~nm}$ is $0.4 \mathrm{~A} / \mathrm{W}$. A $1.8 \mathrm{~mA}$ current will be created in the diode.

Figure $4-4$ is a diagram for the circuit used to create an amplified voltage from the diode current. The voltage output will be

$$
V=G R_{i} i
$$

where $i$ is the diode current, $R_{1}$ is the resistor across the diode as shown in the diagram, and $G$ is the op-amp gain. For the values listed in figure 4-4 the op-amp 


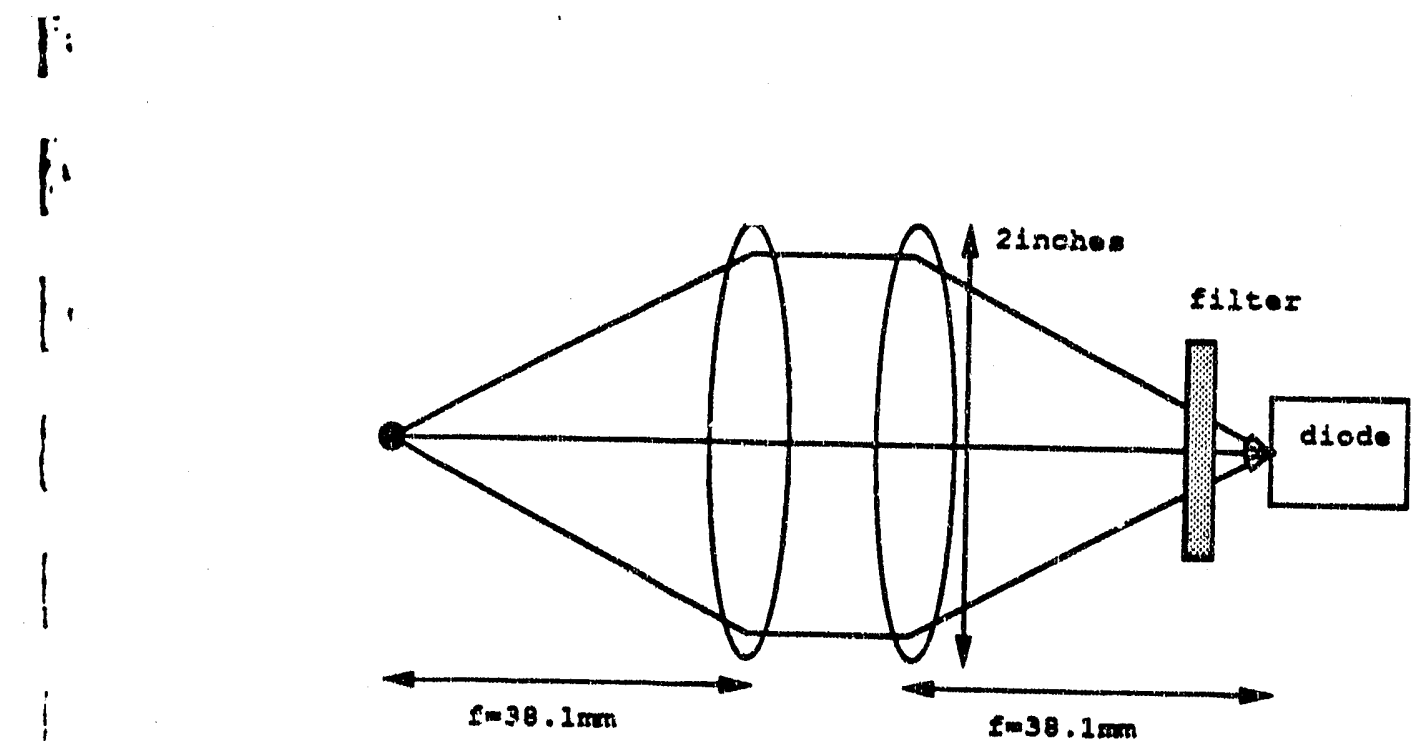

Figure 4-5: Focusing the Fluorescence onto a Single Photodiode

output saturates in room light. Measuring a $1.2 \mathrm{~mA}$ current should be possible. Many of the same assumptions were made as in the calculation for the diode array.

The collection efficiency can be increased, if necessary by focusing the fluorescence light with two lenses as shown in figure 4-5. The collection efficiency of this design is 0.17 for the values in figure $4-5$ because the lenses are much bigger than the diode.

\subsubsection{Normalization}

From shot to shot the energy in the laser pulses fiuctuates. Thus, the autocorrelation signal will be ambiguous unless the measured fluorescence energy is normalized relative to the pulse energy so that the fluctuations do not effect the measurement. Because the fluorescence is proportional to the square of the pulse power, the signal must be normalized by dividing by the square of the pulse power. This signal is proportional to the contrast ratio, $S(\tau) / S(\infty)$. There are two ways of normalizing the signal.

One rnethod is to use another dye cell to measure the fluorescence created by the uncorrelated single - by the signal in one of the interferometer arms or by a signal split off the incoming beam. A telescope containing a dye cell can be put before the interferometer to measure the uncorrelated signal proportional to $S(\infty)$. Appendix $A$ is the detailed circuit diagram and optical design for this scheme. 
The other method is to measure the $1.054 \mu \mathrm{m}$ signal and square it electronically. A design is shown in figure 4-6. Because the second diode is measuring the IR signal, it does not matter that the signal measured is from both arms of the interferometer. The diode will not measure a correlation signal. 


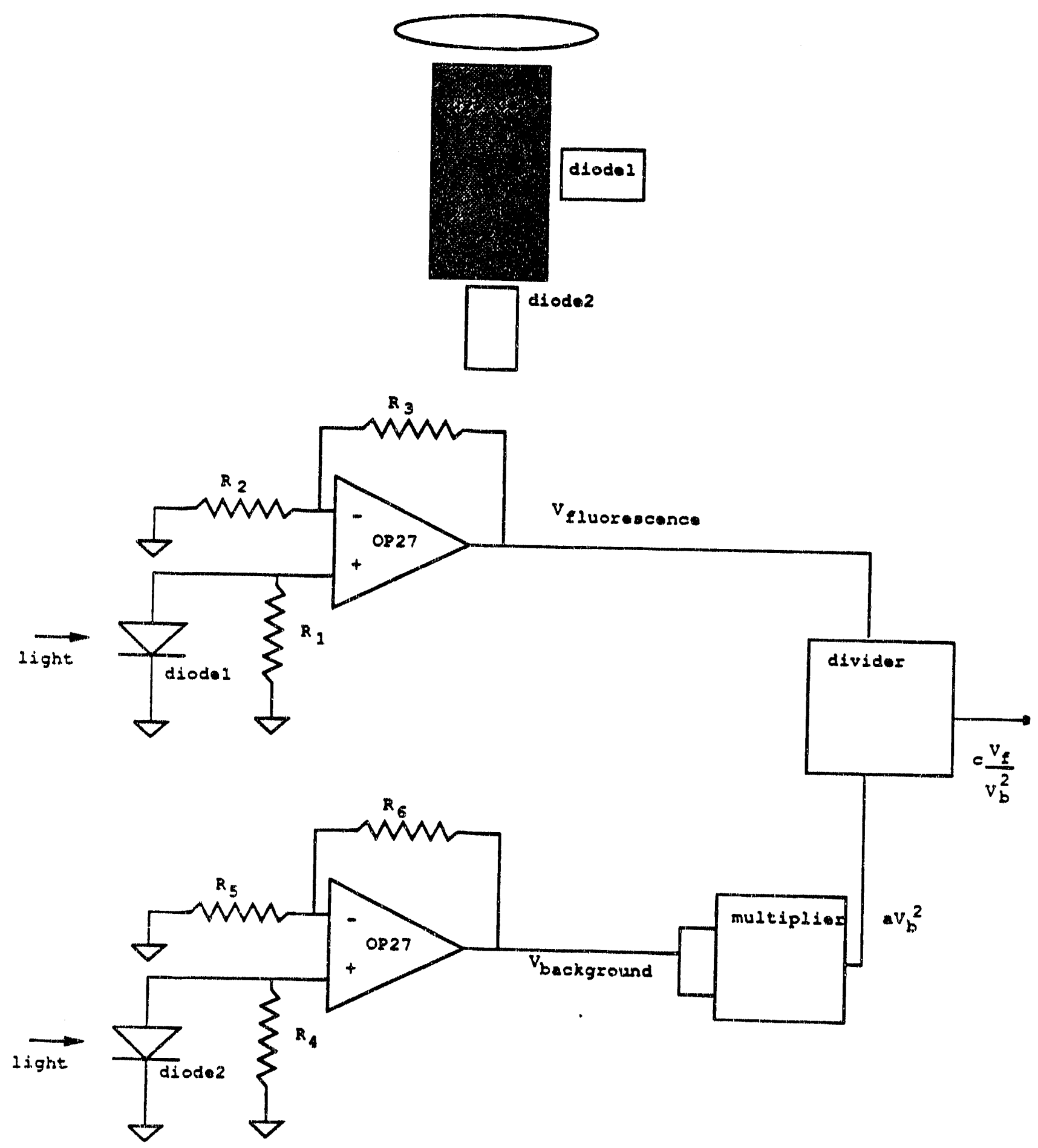

Figure 4-6: A design for electronically normalizing the measured fluorescence signal 


\section{Chapter 5}

\section{Experimental Results}

In this chapter I will discuss the extent and results of my attempts to measure the autocorrelation signal using the methods discussed in the previous chapters. Results of measuring the pulse width are inconclusive. I will discuss what results I did get and explain difficulties I had.

The design in which each pulse is collided with the next pulse in the train was used. Figure 5-1 shows this design again with the parameter values used. Using a $5 \mathrm{~cm}$ dye cell of 0.01 Molarity Rhodamine $6 \mathrm{G}$ in ethanol, the fluorescence trace can be seen with the naked eye. The two lenses were adjusted so that the IR beam leaving the $50 \mathrm{~cm}$ lens did not appear to diverge or focus. The position of the $50 \mathrm{~cm}$ lens was then fine tuned by moving it until the fluorescence trace of the returning beam was the brightest. This was done with the end mirror misaligned so that the arriving and returning beams were separated.

To determine the distance between pulses, the pulse train was measured with a fast photodiode and Tektronix SCD 1000 digitizer with a resolution of $\sim 1$ ns. The pulses were measured to be 7.5ns apart. This corresponds to 2.25 meters separation in air and 2.178 meters separation including travel time through the dye cell. (The index of the dye solution is 1.44.) The distance between the mirror and the center of the dye cell was set to 1.09 meters using a tape measure. The end mirror was aligned by looking at the return beam on an IR card with a hole cut in it for the arriving beam to pass through. The return bearn was aligned so that it also went through the 


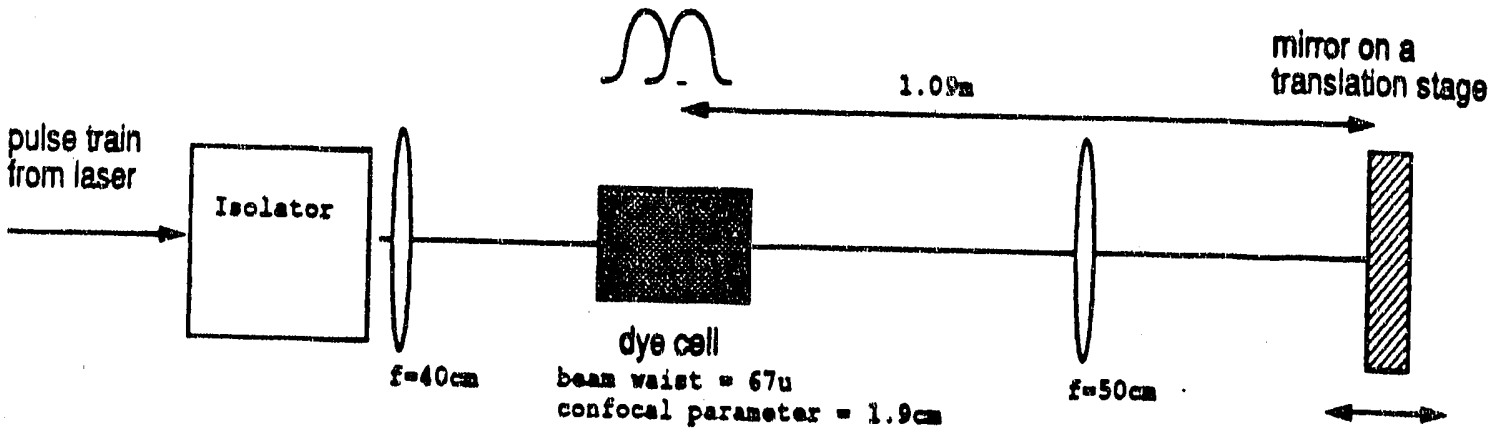

Figure 5-1: Autocorrelator Design used

hole cut in the IR card.

Data was taken with the diode array as close to the fluorescence streak as possible without using any imaging optics. The height of the beam was measured with an IR card and ruler, and then the diode array was set to the same height. The visible signal produced by the IR card is fatter than the IR beam so that there is some uncertainty in this method. Two 65.1150 BG filters were taped in front of the array to block scattered JR. The interference filter mentioned in chapter 4 had not yet arrived. In practice, it was very difficult to adjust and align the imaging optics. When the laser is run continuously, it shoots every 2 seconds, but it did not generate enough fluorescence to find the image. When the laser is shot every 30 seconds using the preamplifier set at 85 , each pulse has $\sim 3 \mathrm{~mJ}$ and there is a lot of fluorescence, but the repetition rate is too low for the lenses to be adjusted in a reasonable amount of time. So, as a first pass, the diode array was set up without any imaging optics. I had intended to return to the problem of lens alignment after the autocorrelation signal had been seen. Then I would have a better idea of what to expect as the correct fluorescence signal.

Figure 5-2 shows an oscilloscope trace measuring the fluorescence with the laser $Q$ switched and modelocked. The preamplifier setting is 85 which corresponds to a shot energy of $\sim 3 \mathrm{~mJ}$. The oscilloscope is triggered from the laser trigger output, and the trace is the video output of the diode array on channel A of the oscilloscope. Figure 5-3 


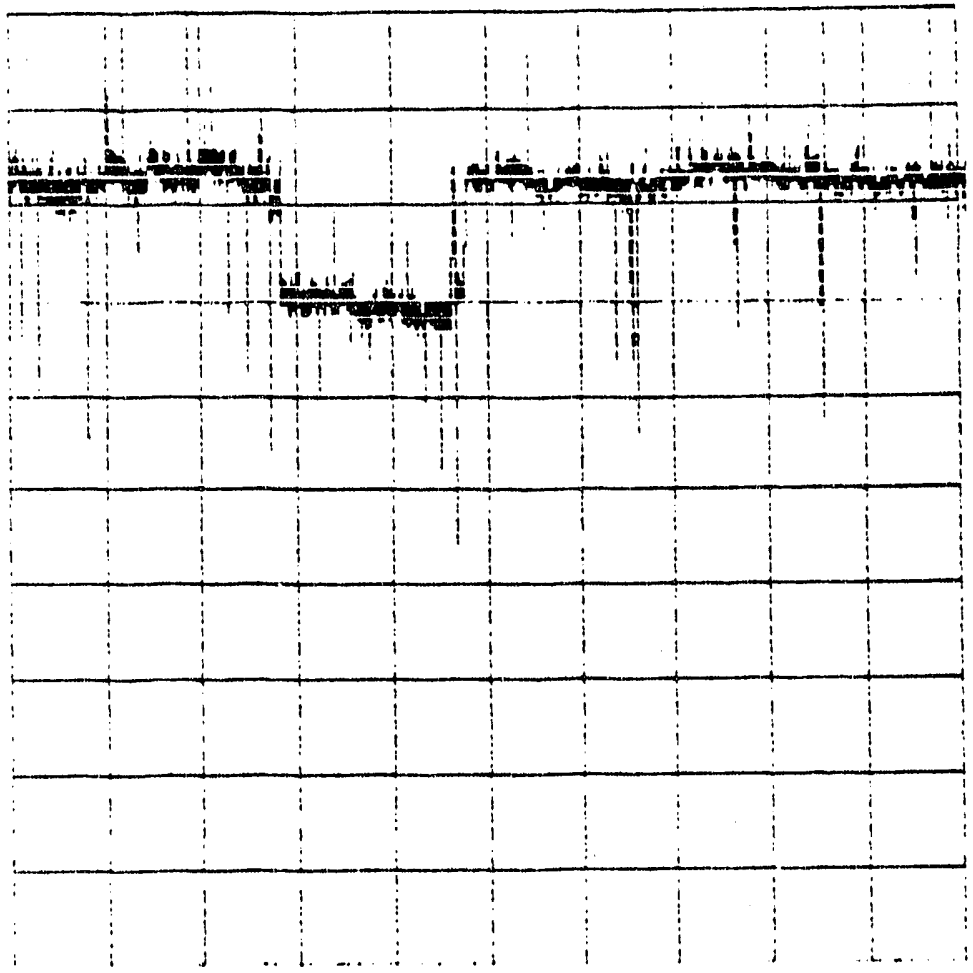

Figure 5-2: oscilloscope trace of array signal with laser Q-switched and modelocked (vertical $10 \mathrm{mV} /$ div, horizontal $1 \mathrm{~ms} / \mathrm{div}$ )

is a trace taken with laser neither Q-switched nor modelocked in order to measure the amount of IR being picked up by the array. As can be seen from this trace no signal can be seen when there is the same amount of IR energy but not enough intensity to generate fluorescence. Thus, the signal measured is the fluorescence signal.

Next. I recorded the fluorescence with the returning beam blocked and unblocked. I was looking for a contrast ratio of close to 6 to 1 as the hallmark of autocorrelation. I varied the position of the end mirror over a few centimeters, recording traces at several different end mirror positions. Figures 5.4 and 5.5 are recorded traces with the beam blocked and unblocked, respectively. Figures 5-6 and 5-7 are traces with the beam blocked and unblocked with the mirror at a different position.

The contrast ratio for the first two traces is 3.8 which corresponds to a measurement of $S(0) / S(\infty)=1.9$. For this measurement, the distance from the mirror to the center of the cell was $\sim 1.08 \mathrm{~m}$. For the second two traces the distance was $\sim 1.07 \mathrm{~m}$. The contrast ratio for the second two traces is 2.28 which corresponds to a mea- 


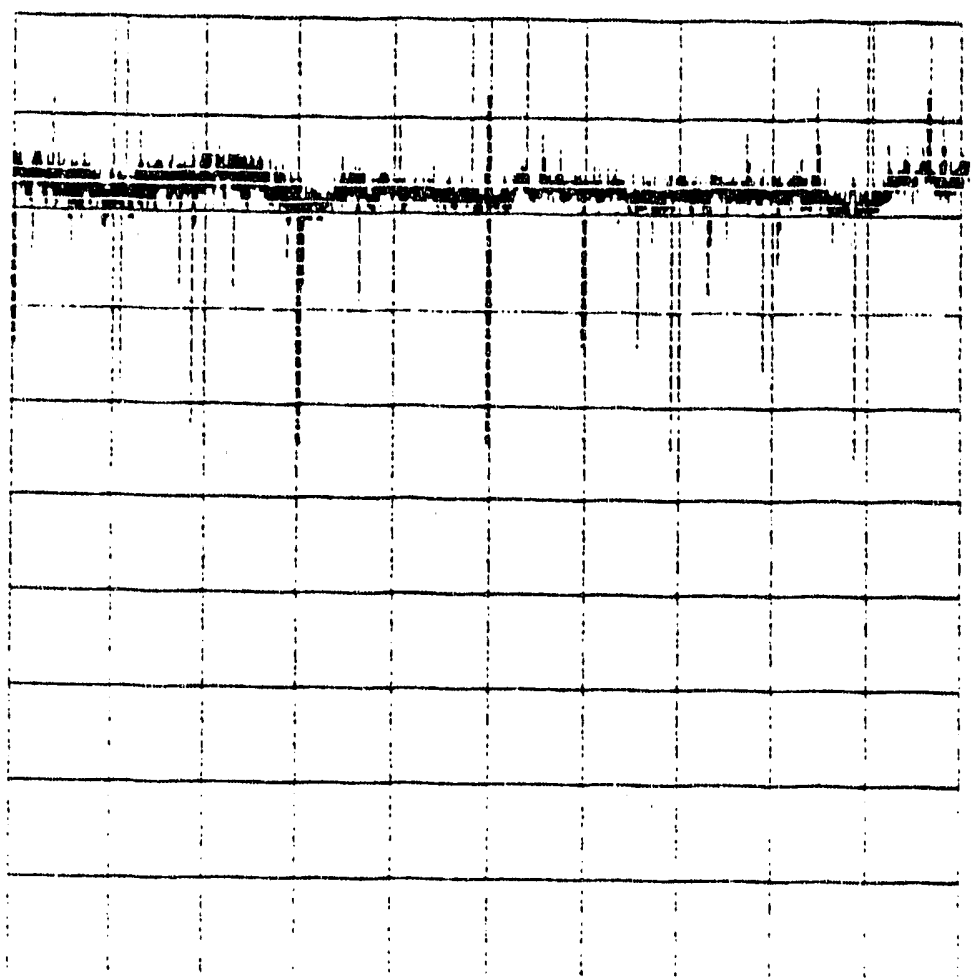

Figure 5-3: oscilloscope trace of array signal with laser neither Q-switched nor modelocked (vertical $10 \mathrm{mV} / \mathrm{div}$, horizontal $1 \mathrm{~ms} / \mathrm{div}$ )

surement of $S(0) / S(\infty)=1.14$. Contrast ratios as low as 1.7 have been measured on ND:glass laser systems by some authors [3], but 1.14 is certainly a very low value. Perhaps the beams were imperfectly aligned in the cell. Fiuctuations in the pulse energy from shot to shot make interpretations of the contrast ratio difficult.

Figure 5-8 compares the two measured contrast ratios with the predicted contrast ratio as a function of the distance of the fluorescence from the end mirror. Curve 1 is the ideal case for which there is no attenuation and the returning pulse has the same intensity of the incoming pulse, $I_{1}$. Curve 2 assumes that the intensity of the returning pulse is $80 \%$ of the intensity of the incoming pulse. This accounts for reflections from the lenses and dye cell. The loss in the dye cell due to absorption is small (2.4\%). Curve 3 accounts for the beam divergence as calculated in equation 2.37 and assumes that the intensity of the returning pulse is $80 \%$ that of the incoming pulse. The time Ops corresponds to a distance of $1.09 \mathrm{~m}$ between the end mirror and the fluorescence section. The distance value for each data point is taken to be the point along the 


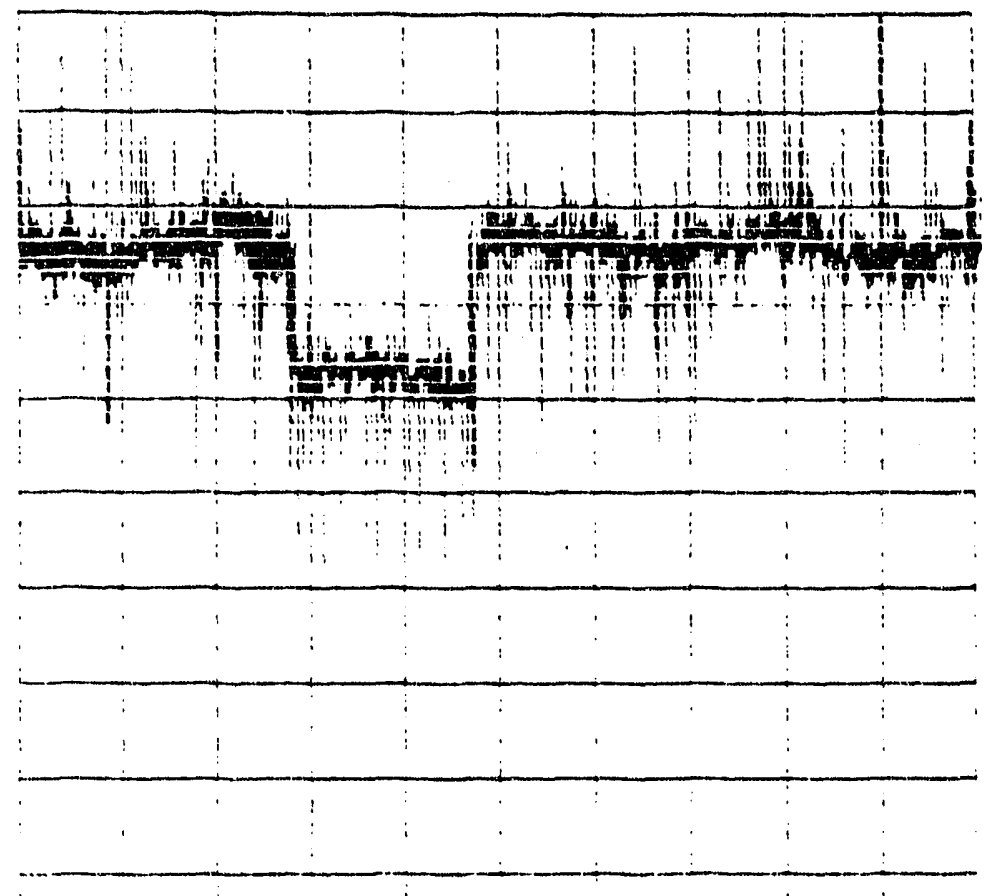

Figure 5-4: fluorescence trace with the beam blocked (vertical $20 \mathrm{mV} /$ div, horizontal $1 \mathrm{~ms} / \mathrm{div}$ ) 
$\because$

F.

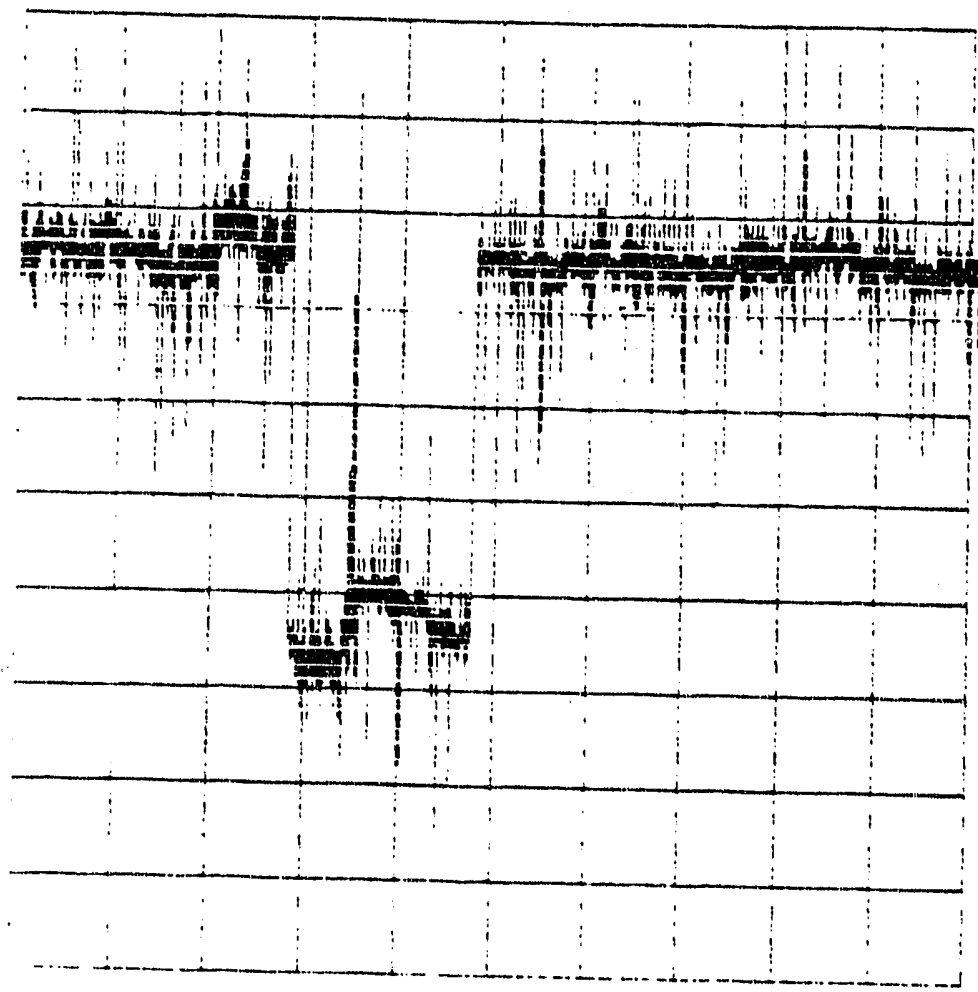
Figure 5-5: fluorescence trace with the beam unblocked (vertical $20 \mathrm{mV} /$ div, horizon-
tal $1 \mathrm{~ms} / \mathrm{div}$ ) 


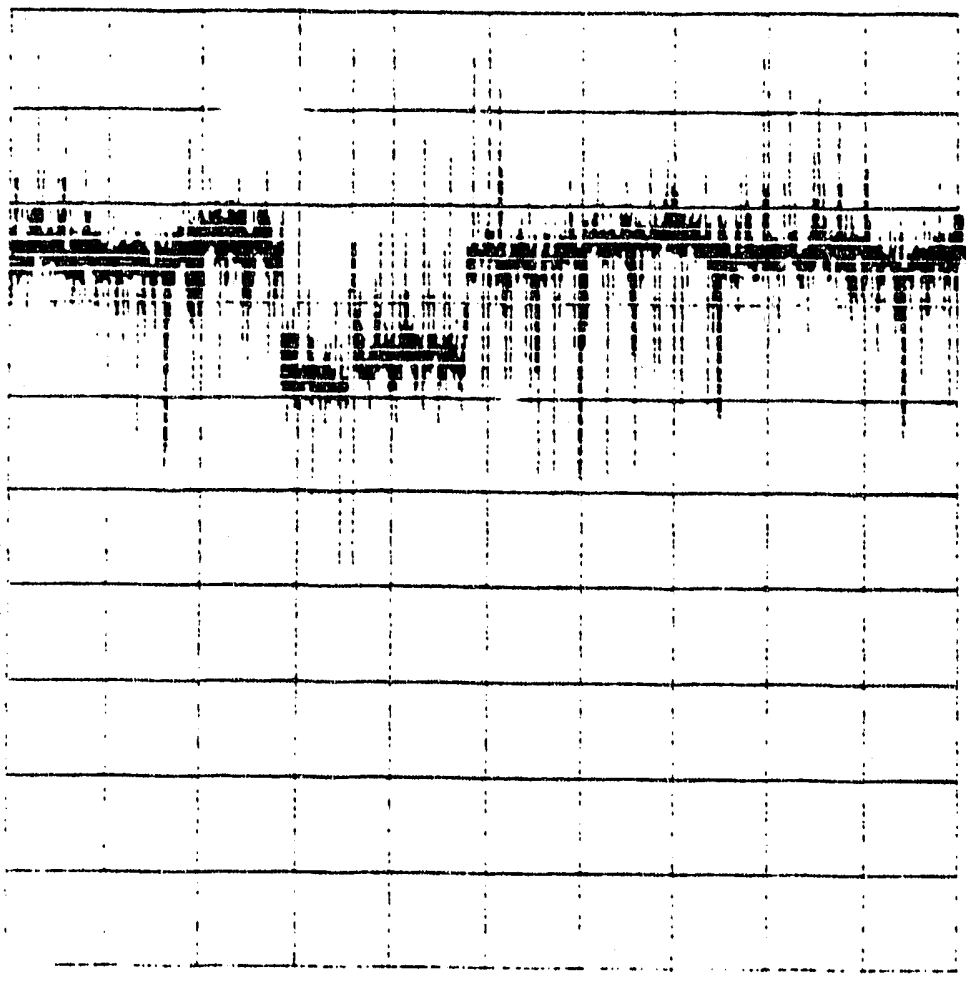

Figure 5-6: fluorescence trace with the beam blocked (vertical $20 \mathrm{mV} /$ div, horizontal $1 \mathrm{~ms} / \mathrm{div}$ ) 


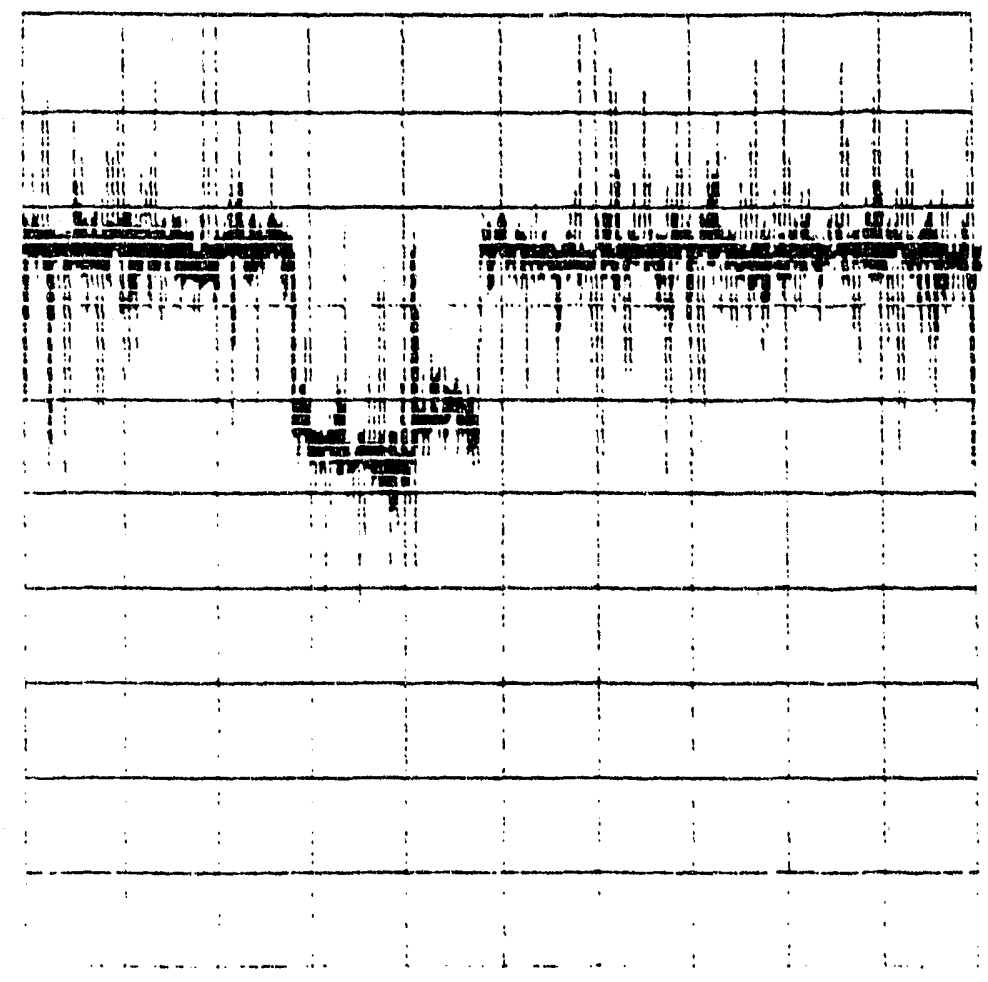

Figure 5-7: fluorescence trace with the beam unblocked (vertical $20 \mathrm{mV} /$ div, horizontal $1 \mathrm{~ms} / \mathrm{div}$ ) 


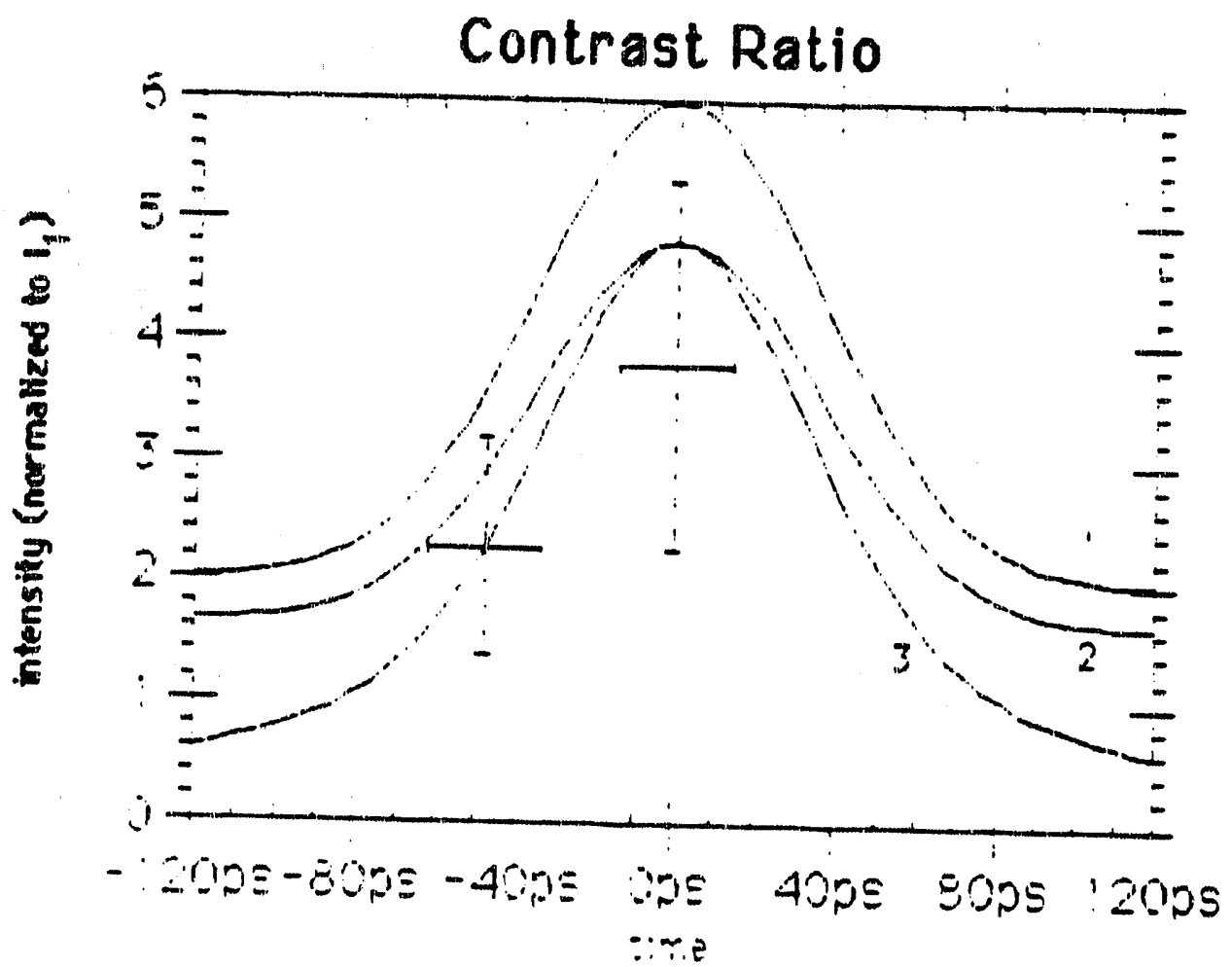

Figure 5.8: Comparison of the predicted contrast ratio to the measured contrast ratio as a function of the distance from the end mirror to the fluorescence: curve 1 is the case with equal beam intensities and no attenuation, curve 2 assumes that the intensity of the returning pulse is $80 \%$ that of the incoming pulse, $I_{1}$, and curve 3 accounts for beam divergence as well.

array where the maximum fluorescence is expected because if the peak fluorescence is anywhere along the length of the aray, it should be detected; the error is estimated from the uncertainty in the position measurement of the end mirror and of the array. The error in intensity is estimated to be $40 \%$ from the fluctuations in pulse energy from shot to shot; the laser was shot many times at the same preamplifier setting and the energy was measured for each shot.

One positive sign is that the fluorescence traces with the returning beam unblocked show much more structure than the traces with the returning beam blocked, which is a sign of autocorrelation. But I also recorded some fluorescence traces with the returning beam blocked which showed some structure. The scan rate of the $1 \mathrm{~cm}$ diode array is $1.92 \mathrm{~ms}$ so that $1 \mathrm{~cm}$ corresponds to time of $1.92 \mathrm{~ms}$ on the ascilloscope trace. Figures 5.4.5.5, 5.6 and 5.7 were taken with the diode array scan not synchronous 
with the oscilloscope scan which was triggered with the laser trigger output.

The results are difficult to interpret which is one of the difficulties with the TPF method as Bradley and New point out [3]. The short length of the dye cell and the diode array make interpreting the results difficult because $S(0)$ and $S(\infty)$ cannot be simultaneously measured. The dye cell is $5 \mathrm{~cm}$ and the width of the autocorrelation signal for a $100 \mathrm{ps}$ pulse is $2.9 \mathrm{~cm}$. Absorption, beam divergence and self-focusing effects make use of longer dye cells difficult [3].

These results are certainly not very satisfying. Unfortunately the laser rod had become damaged over the past several months, so that the laser performance was becoming steadily worse. Next, I plan to build and test the copropagating pulse design. This design is more sensitive and easier to align, so I hope for more positive results. 


\section{Chapter 6}

\section{Summary and Conclusions}

The theory of TPF autocorrelation was explained and different designs were proposed. Designs for imaging the TPF trace on a diode array were evaluated. While it appears that imaging on a diode array is feasible, experiments indicated that the measured signal is less than expected.

TPF autocorrelation relies on the nonlinearity of two-photon absorption by the dye solution to create a fluorescence signal proportional to the square of the laser intensity. If the laser pulses are split and collided with themselves in the dye solution, the time integrated signal recorded by a detector will be the autocorrelation of the signal as a function of distance.

In the ideal case, the contrast ratio between the fluorescence at the point of max. imum temporal pulse overlap and the fluorescence with no pulse overlap should be 3 to 1. But the contrast ratio will be reduced if the pulse intensities are unequal and if the spatial overlap of the pulses is imperfect. In practice, it is difficult to make the pulse intensities equal and to make the spatial overlap perfect.

The traditional design is a triangular configuration in which the pulse from the laser is split with a $50 \%$ beam splitter and each of the resulting pulses is guided with a mirror into one end of the dye cell. The disadvantage of this design is that most $30 \%$ beam splitters are polarization sensitive so that a cheap simple isolator cannot be used. Isolation proved to be an important issue. The limit that isolation puts on the pulse energy that can be used is a significant constraint on the system. With 
higher pulse energies, more fluorescence power can be produced with less concern for beam divergence and focusing problems.

In another configuration, a mirror can be put at the end of the dye cell or immersed in the dye so that the front of each pulse can be correlated with the end. In this design care must be taken not to damage the mirror if the beam is tightly focused, but this design is less sensitive to alignment than others.

Each pulse can also be correlated with its nearest neighbor in the pulse train by reflecting the pulses with a mirror positioned $1.09 \mathrm{~m}$ - for the ND:YLF -from the dye cell so that the pulses collide in the cell. In this design, the pulses can be focused into the dye cell without damaging the optics, and there are no polarization sensitive elements. Experiments were made with this design.

To image the fluorescence from the whole dye cell on to a diode array the fluorescence must be demagnified by 5. A design using two 2 inch lenses was examined. A simple approximate calculation indicated that $0.2 \mathrm{~mJ} /$ shot would saturate the array near the center where the collection efficiency is the largest. A similar calculation indicated that $2.3 \mathrm{~mJ} / \mathrm{shot}$ would saturate the array without any imaging optics. It was not expected that the array would, in fact, saturate because of the simplifications made in the calculations. The alignment of the array is important because both the array and the fluorescence streak are very narrow. Without using imaging optics, a $\sim 40 \mathrm{mV}$ signal was measured with a laser energy of $\sim 3 \mathrm{~mJ} /$ shot; saturation occurs at $2.5 \mathrm{~V}$.

The experimental results are difficult to interpret for several reasons. Because of fluctuations in the laser energy from shot to shot, the calculated contrast is not very accurate and varied from trial to trial. The contrast ratio is reduced both by imperfect beam alignment and unequal beam intensities so that it is unclear whether a low contrast ratio is due to the laser or these factors. The theoretical contrast satio for aoise is 1.5 so that contrast ratios consistently higher than this must be measured to insure that the autocorrelation of modelocked pulses is being measured.

The measurements did exhibit structure when the colliding pulses were measured, indicating that pulse structure was being measured. When the fluorescence of only 
one pulse was measured, the signal was flat.

An important difficulty with the system is the alignment of the both the pulses and the imaging system. The alignment of the pulses can be done fairiy well using a Helium-Neon laser to align the optics, but the alignment of the imaging system requires using the ND:YLF to create fluorescence. The alignment is critical because both the fluorescence streak and the diode array are narrow, and the low repetition rate of the laser makes alignment both tedious and difficult. The signal measured on the diode array with no imaging optics was significantly less than the expected signal calculated from the equations in section 4.2. Either the alignment was off or the calculation needs to be reevaluated.

A design was also considered in which a Michelson interferometer was used to vary the delay between the two copies of the pulse. The two copies are recombined and focused into the dye cell where the signal is measured with a large area diode. This design is more sensitive than the others, but it requires several shots to measure the autocorrelation.

For future work, I would recommend that film or a video system be on hand for measuring the autocorrelation signal. Although the intention of the design described in this paper is that the signal be recorded with a diode array, using film would allow one to verify that the alignment of the pulses was correct, and the amount of energy being recorded could be measured. Difficulties would be easier to locate. Measurements of TPF autocorrelation signals with film are well documented in the literature. TPF autocorrelators can provide important information about modelocked lasers. The use of diode arrays to measure the signal requires further evaluation. 


\section{Appendix A}

\section{Optical design and circuit diagram for section 4.3.1}

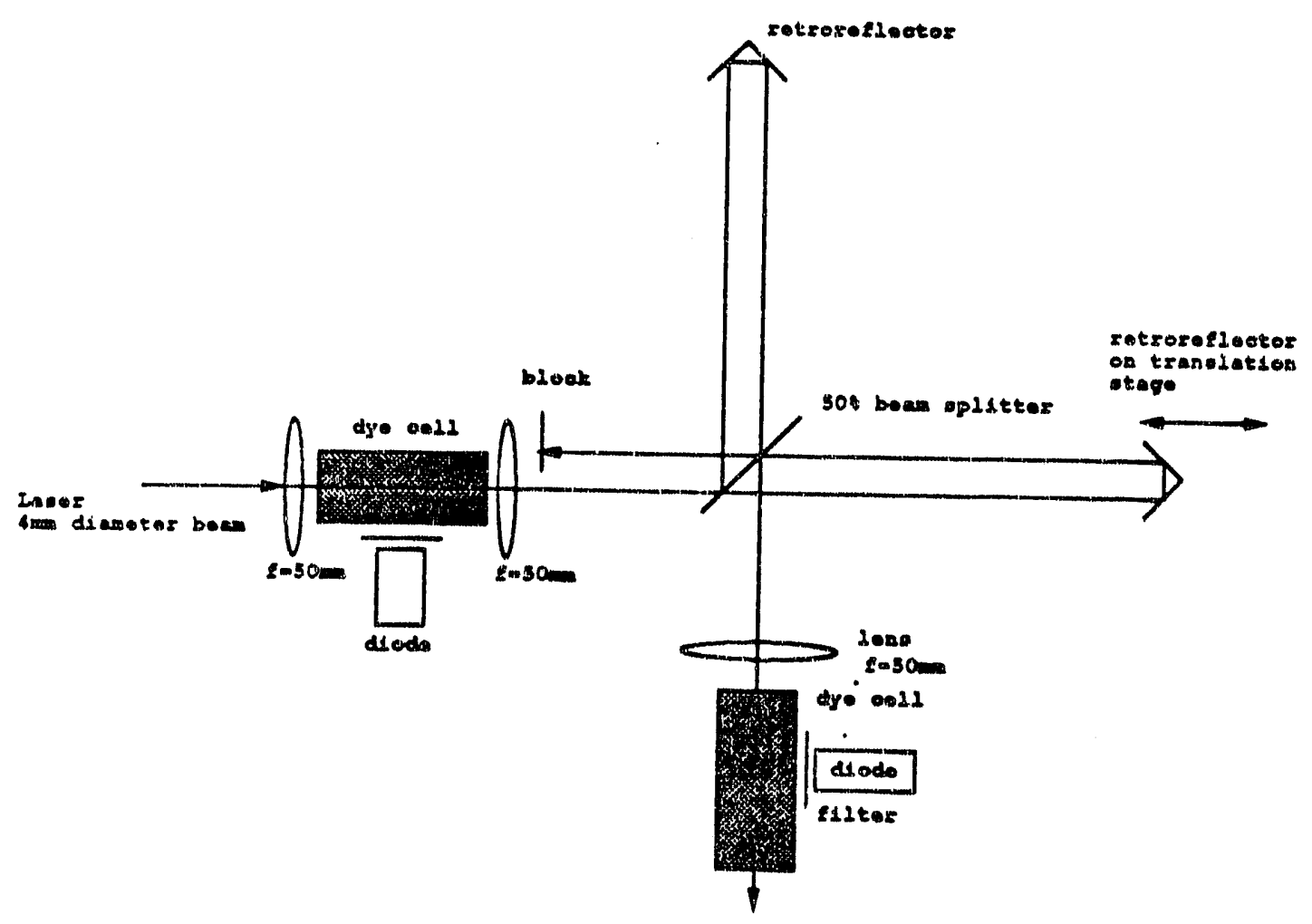




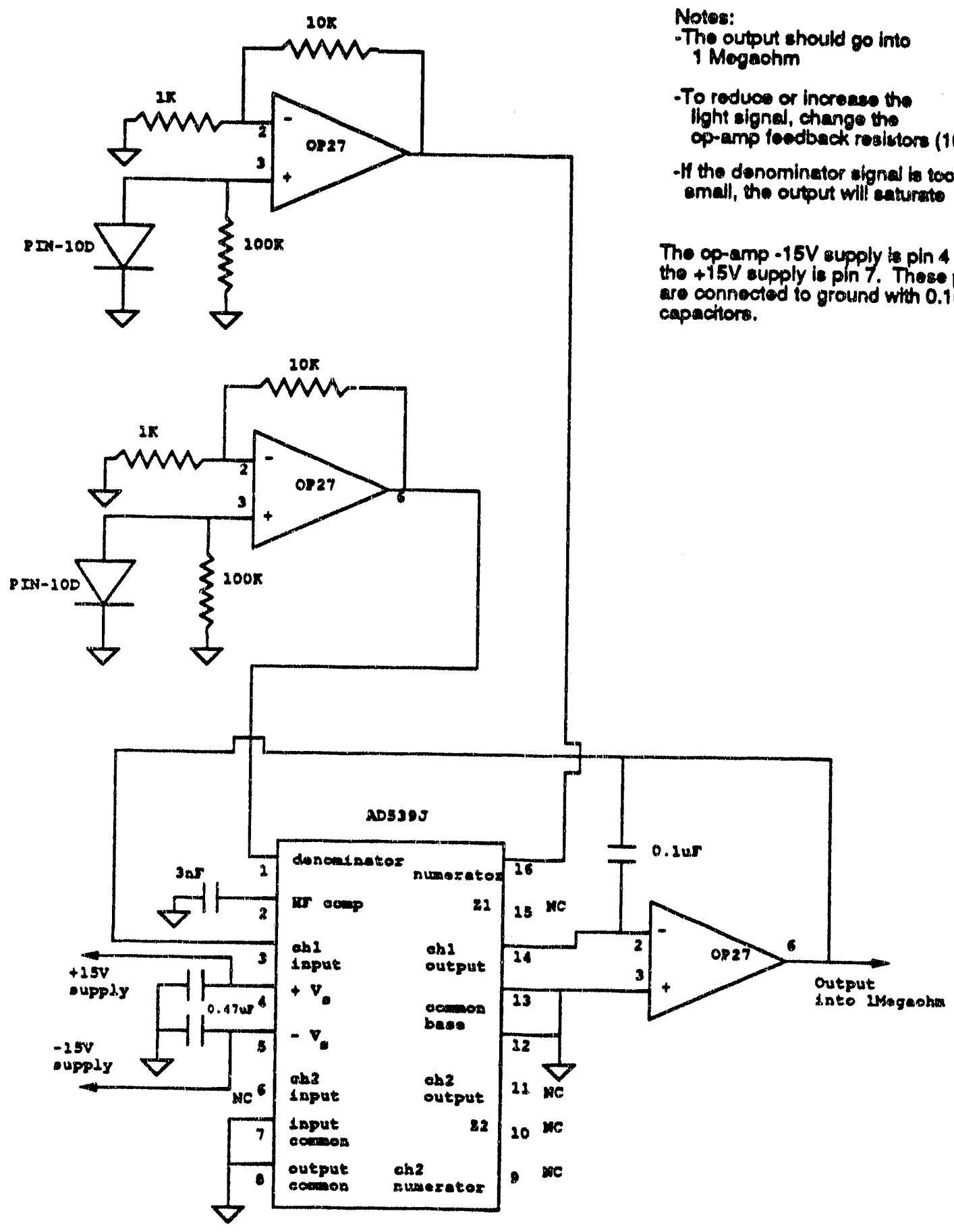




\section{Bibliography}

[1] J. H. Bechtel and Smith W. L. Two-photon fluorescence in attenuating media and laser pulse duration measurements. Journal of Applied Physics, 46(11), November 1975.

[2] D. J. Bradley. Measurement of two-photon and single-photon absorption cross sections and picosecond relaxation times in organic dyes. IEEE Journal of Quantum Electronics, QE-8(6), June 1972.

[3] D. J. Bradley and Geoffrey H. C. New. Ultrashort pulse measurements. Proceedings of the IEEE, 62(3), March 1974.

[4] Shapiro S. L. Giordmaine J. A., Rentzepis P. M. and Wecht K. W. Two-photon excitation of fluorescence by picosecond light pulses. Applied Physics Letters, $11(7), 1967$.

[5] Hermann A. Haus. Waves and Fields in Optoelectronics. Prentice-Hall, Inc., Englewood Cliffs, NJ, 1984.

[6] Erich P. Ippen. Nonlinear optics. Course notes for MIT course 6.634, 1991.

[7] Richard H. Pantell and Harold E. Puthoff. Fundamentals of Quantum Electron. ics. John Wiley and Sons, New York, NY, 1969.

[8] F. P. Schafer, editor. Dye Lasers, Srd ed. Springer-Verlag, 1990.

[9] P. Sperber and A. Penzkofer. Pulse-shape determination of intracavity compressed picosecond pulses by two-photon fluorescence analysis. Optical and Quantum Electronics, 18, 1986. 
[10] Rolf A. Wyss. Single pulse measurement using autocorrelation. written for MIT optics lab, January 1990. 

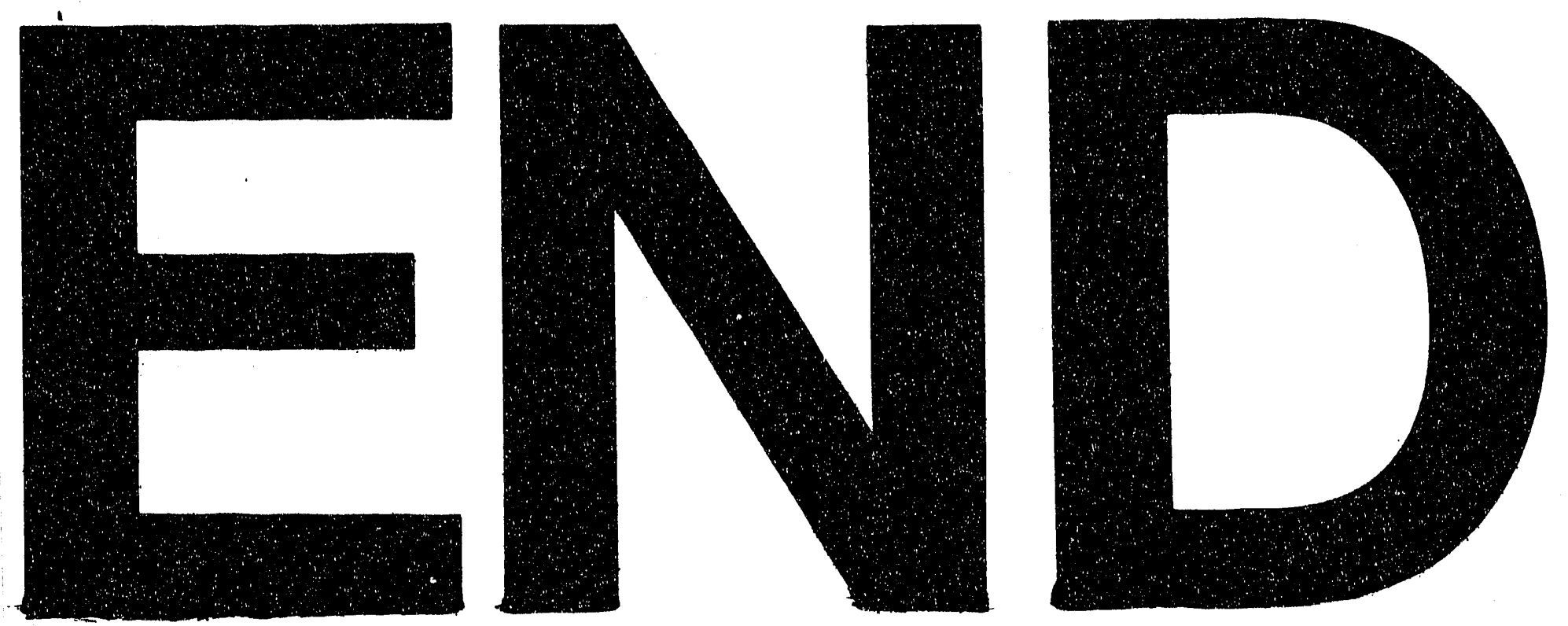

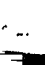

4
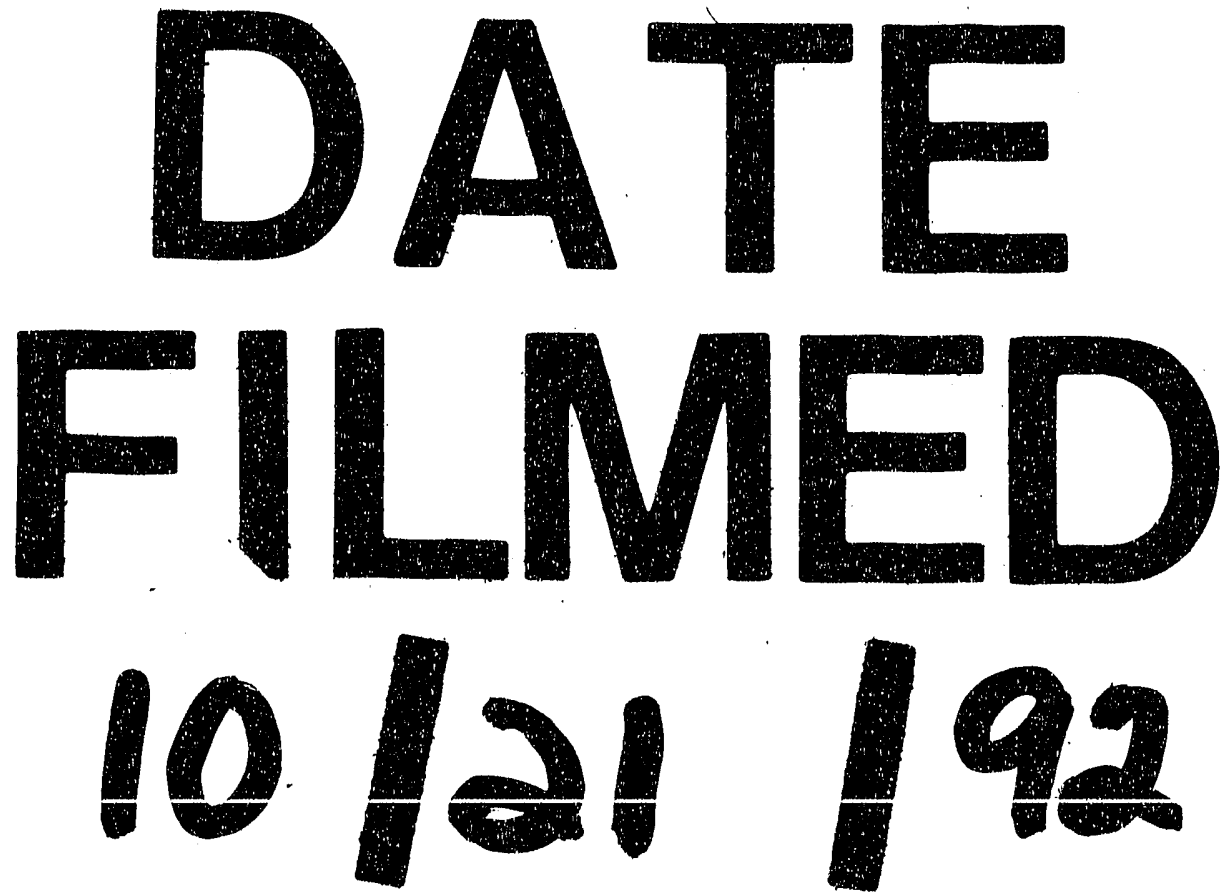
